\title{
NEW RECORDS OF LEPIDOPTERA (INSECTA) OF SEVERODONETSK (LUHANSK REGION, UKRAINE) AND ITS ENVIRONS
}

\begin{abstract}
Дем'яненко, С. О., Бідзіля, О. В., Каролінський, С. О. Нові знахідки лускокрилих (Insecta: Lepidoptera) Сєвєродонецька (Луганська область, Україна) та його околиць. Вісті Харківського ентомологічного товариства. 2021. Т. ХХІХ, вип. 1. C. 20-52. DOI: 10.36016/KhESG-2021-29-1-3.

Наведено анотований список нових знахідок лускокрилих Сєвєродонецька. Список містить 425 нових для Сєвєродонецька видів, 3 яких 190 видів уперше вказані для території Луганської області, а 1 вид (Ethmia vittalbella (Christoph, 1877)) - уперше для України. Загалом у Сєвєродонецьку відмічено 1074 види Lepidoptera. 1 таб., 36 рис., 31 назв.
\end{abstract}

Ключові слова: Lepidoptera, лускокрилі, фауна, Сєвєродонецьк, Луганська область.

Демьяненко, С. А., Бидзиля, А. В., Каролинский, Е. А. Новые находки чешуекрылых (Insecta: Lepidoptera) Северодонецка (Луганская область, Украина) и его окрестностей. Известия Харьковского энтомологического общества. 2021. Т. ХХІХ, выI. 1. C. 20-52. DOI: 10.36016/KhESG-2021-29-1-3.

Приводится аннотированный список новых находок чешуекрылых Северодонецка. Список включает в себя 425 новых для Северодонецка видов, из которых 190 видов впервые указаны для Луганской области, а 1 вид (Ethmia vittalbella (Christoph, 1877)) — впервые для фауны Украины. Всего в Северодонецке отмечены 1074 вида Lepidoptera.

Ключевые слова: Lepidoptera, чешуекрылые, фауна, Северодонецк, Луганская область.

Demyanenko, S. O., Bidzilya, O. V., Karolinskiy, E. A. New records of Lepidoptera (Insecta) of Severodonetsk (Luhansk Region, Ukraine) and its environs. The Kharkov Entomological Society Gazette. 2021. Vol. XXIX, iss. 1. P. 20-52. DOI: 10.36016/KhESG-202129-1-3.

An annotated list of new records of butterflies and moths of Severodonetsk is provided. The list includes 425 species registered in Severodonetsk for the first time, 190 species of which are new records for Luhansk Region, and 1 species (Ethmia vittalbella (Christoph, 1877)) is new for Ukraine. Totally, there are 1,074 species of Lepidoptera registered in Severodonetsk. 1 tab., 36 figs, 31 refs. Keywords: Lepidoptera, butterflies and moths, fauna, Severodonetsk, Luhansk Region.

I n trodu ct i o n. Previously data on Lepidoptera of Severodonetsk and its environs appeared in the publications shown in Table 1. We exclude three species, Scopula nemoraria (Hübner, [1799]), Cidaria fulvata (Forster, 1771) (Demyanenko, Severov, Kostjuk, 2011) and Sciota hostilis (Stephens, 1834) (Demyanenko, 2019), from the list of Severodonetsk and Lugansk region due to misidentification of Scopula floslactata (Haworth, [1809]), Eulithis mellinata (Fabricius, 1787) and Apomyelois bistriatella (Hulst, 1887) respectively. Totally, 649 species of Lepidoptera were known from Severodonetsk prior to this work.

Materials a nd methods. This paper is based on the material collected and observed by the first author (otherwise the name of collector is indicated) in Severodonetsk and its close environs since 1996.

The material was observed, photographed, and collected by netting during daytime excursions, attracting by light (160 W and $250 \mathrm{~W}$ mercury-vapor lamps), and in some cases by attracting by wine lure.

The determination of the material was carried out by the authors. The identification by photographs was made only for species with distinct differences in habitus. In difficult cases, the determination was made by genitalia prepared by maceration in $8-12 \% \mathrm{NaOH}$ solution. Determination of mines were made by Ellis (2021) and Lepiforum (2020). Plant names are given by 'Plants of the World Online' (POWO, 2021).

The material is stored in the collections of the Institute for Evolutionary Ecology of the National Academy of Sciences of Ukraine (Kyiv) and the Museum of Nature of the Vasyl Karazin Kharkiv National University.

The main place for attracting by light is $5 \mathrm{~km} \mathrm{~S}$ Severodonetsk, 'dacha' area $\left(48.904^{\circ} \mathrm{N}, 38.509^{\circ} \mathrm{E}\right)$, further in text 'D' (in 2007-2012 we used mercury-fluorescent $250 \mathrm{~W}$ arc lamp, in 2013-2016 - mercurytungsten $250 \mathrm{~W}$ arc lamp, after 2016 - mercury-tungsten $160 \mathrm{~W}$ arc lamp, unless stated otherwise). Besides this, we recorded a few specimens of Lepidoptera attracted by incandescent and LED lamps within the city $\left(48.934^{\circ} \mathrm{N}, 38.494^{\circ} \mathrm{E}\right)$, further in text ' $\mathrm{C}$ '. In addition, single specimens in both of the above-mentioned locations were registered in the daytime (in such cases it is indicated in the text).

Demyanenko S. O. Severodonetsk, Luhansk Region, 93400, UKRAINE; e-mail: severlepsd@gmail.com; ORCID: 0000-0002-7915-2546 Bidzilya $\boldsymbol{O}$. V. Institute for Evolutionary Ecology of the National Academy of Sciences of Ukraine,

37, Lebedeva St., Kyiv, 03143, UKRAINE; e-mail: olexbidzilya@gmail.com, ORCID: 0000-0001-9243-2481 Karolinskiy E. A. Vasyl Karazin Kharkiv National University,

4, Svobody Sqr., Kharkiv, 61022, UKRAINE; e-mail: kharkov.but@gmail.com; ORCID: 0000-0002-1109-4247 
S. O. DEMYANENKO, O. V. BIDZILYA, E. A. KAROLINSKIY New records of Lepidoptera (Insecta) of Severodonetsk (Luhansk Region, Ukraine) and its environs

Ta b l e 1. Publications on Lepidoptera of Severodonetsk and its environs

\begin{tabular}{|c|c|c|c|c|c|c|c|c|c|c|c|c|c|c|c|c|c|c|c|c|c|c|c|c|c|c|}
\hline \multirow[b]{2}{*}{ Publications } & \multicolumn{26}{|c|}{ Number of species by family } \\
\hline & 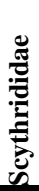 & 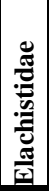 & 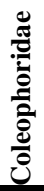 & 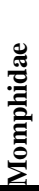 & 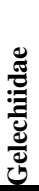 & 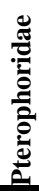 & 苞 & 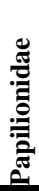 & 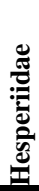 & 䒕 & 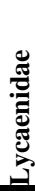 & 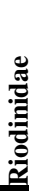 & 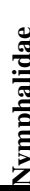 & & & & 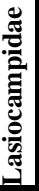 & 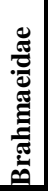 & 窇 & 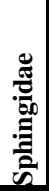 & 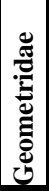 & 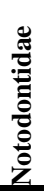 & 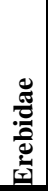 & 莺 & 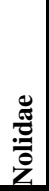 & 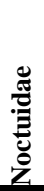 \\
\hline Bidzilya, 1994 (1995) & - & - & - & - & - & 1 & - & - & - & - & - & - & - & - & - & & - & - & - & - & - & - & - & - & - & - \\
\hline Pak, Yaroshenko, 2001 & - & - & - & - & - & - & - & - & - & - & - & - & - & 5 & 2 & & - & - & - & - & - & - & - & - & - & - \\
\hline $\begin{array}{l}\text { Klyuchko, Severov, } \\
2006\end{array}$ & - & - & - & - & - & - & - & - & - & - & - & - & - & - & - & & - & - & - & 1 & - & - & 1 & - & - & 2 \\
\hline $\begin{array}{l}\text { Klyuchko, Matov, } \\
\text { Severov, } 2006\end{array}$ & - & - & - & - & - & - & - & - & - & - & - & - & - & - & - & & - & - & - & - & - & - & 12 & 1 & 2 & 58 \\
\hline $\begin{array}{l}\text { Demyanenko, Severov, } \\
\text { Kostjuk, } 2011\end{array}$ & - & - & - & - & - & - & - & - & - & - & - & - & - & - & - & & - & - & - & - & 124 & - & - & - & - & - \\
\hline Geryak et al., 2012 & - & - & - & - & - & - & - & - & - & - & - & - & - & - & _ & & - & - & - & - & - & 5 & 19 & - & 2 & 44 \\
\hline Demyanenko, 2012 & - & - & - & - & - & - & - & - & - & - & - & - & - & - & - & & 2 & 1 & 1 & 14 & - & - & - & - & - & - \\
\hline $\begin{array}{l}\text { Demyanenko, Geryak, } \\
2012\end{array}$ & - & - & - & - & - & - & - & 3 & 6 & 11 & 21 & 1 & 27 & - & - & & - & - & - & - & - & - & - & - & - & - \\
\hline Bidzilya et al., 2013 & - & - & - & - & - & - & - & - & - & - & - & - & - & 1 & 1 & & - & - & - & - & - & - & - & - & - & - \\
\hline $\begin{array}{l}\text { Budashkin, Bidzilya, } \\
\text { Zhakov, } 2014\end{array}$ & - & - & 4 & - & - & - & - & - & - & - & - & - & - & - & - & & - & - & - & - & - & - & - & - & - & - \\
\hline Bidzilya et al., 2014 & - & - & - & 1 & - & - & 2 & - & - & - & - & - & - & - & - & & - & - & - & - & - & - & - & - & - & - \\
\hline Geryak et al., 2014 & - & - & - & - & - & - & - & - & - & - & - & - & - & - & - & & - & - & - & - & - & - & - & - & 1 & - \\
\hline Geryak et al., 2015 & - & - & - & - & - & - & - & - & - & - & - & - & - & - & - & & - & - & - & - & - & 2 & 15 & - & - & 47 \\
\hline Guglya, 2015 & - & - & - & - & - & - & 1 & - & - & - & - & - & - & - & - & & - & - & - & - & - & - & - & - & - & - \\
\hline $\begin{array}{l}\text { Bidzilya, Budashkin, } \\
\text { Zhakov, } 2016\end{array}$ & - & 1 & - & - & - & - & - & - & - & - & - & - & - & - & - & & - & - & - & - & - & - & - & - & - & - \\
\hline $\begin{array}{l}\text { Bidzilya, Budashkin, } \\
2017\end{array}$ & - & - & - & - & 1 & - & - & - & - & - & - & - & - & 1 & - & & - & - & - & - & - & - & - & - & - & - \\
\hline $\begin{array}{l}\text { Bidzilya, Budashkin, } \\
\text { Zhakov, } 2017\end{array}$ & 4 & - & - & - & - & - & - & - & - & - & - & - & - & - & - & & - & - & - & - & - & - & - & - & - & - \\
\hline Geryak et al., 2018 & - & - & - & - & - & - & - & - & - & - & - & - & - & - & - & & - & - & - & - & - & - & 7 & - & 1 & 16 \\
\hline Demyanenko, 2018 & - & - & - & - & - & - & - & - & - & - & - & - & - & - & - & & - & - & - & - & - & 11 & 32 & - & 2 & 101 \\
\hline Kavurka et al., 2018 & - & - & - & - & - & - & - & 2 & - & - & - & 1 & 1 & - & - & & - & - & 1 & - & - & - & 1 & - & - & 1 \\
\hline $\begin{array}{l}\text { Budashkin, Bidzilya, } \\
\text { Demyanenko, } 2019\end{array}$ & - & - & 35 & - & - & - & - & - & - & - & - & - & - & - & - & & - & - & - & - & - & - & - & - & - & - \\
\hline Demyanenko, 2019 & - & - & - & - & - & - & - & - & - & - & - & - & - & - & 7 & & - & - & - & - & - & - & - & - & - & - \\
\hline $\begin{array}{l}\text { Bidzilya, Budashkin, } \\
\text { Yepishin, } 2020\end{array}$ & - & - & - & - & - & - & - & - & - & - & - & - & - & & 2 & & - & - & - & - & - & - & - & - & - & - \\
\hline
\end{tabular}

Daytime excursions were made to the floodplain forest area (mainly oak-maple) SW Severodonetsk $\left(48.91^{\circ} \mathrm{N}, 38.47-38.50^{\circ} \mathrm{E}\right)$ - further in text ' $\mathbf{L}$ ', and to sandy-steppe areas in the pine forest (with alder-birch forests and oak plantings) SE, NE, and $\mathrm{N}$ of Severodonetsk $\left(48.91-48.92^{\circ} \mathrm{N}\right.$ and $38.49-38.65^{\circ} \mathrm{E}, 48.95-48.97^{\circ} \mathrm{N}$ and $38.54-38.60^{\circ} \mathrm{E}, 49.00-49.02^{\circ} \mathrm{N}$ and $\left.38.49-38.52^{\circ} \mathrm{E}\right)$, - further in text ' $\mathbf{P}$ '.

In the list below all species are new for Severodonetsk, an asterisk $(*)$ indicates a species new for Luhansk Region; two asterisks (**) — a species new for Ukraine. For the species new for Ukraine, its general distribution is given. (2014).

The higher system of Lepidoptera follows Nieukerken et al. (2011) with corrections from Heikkilä et al.

Results.

Infraorder D A C N O N Y P H A Hinton, 1946

Superfamily ER I O C R A N I O I D A Rebel, 1901

F amily ER I O C R A N I D A E Rebel, 1901

* Dyseriocrania subpurpurella (Haworth, 1828)

M a t e r i a l. D: 29.04.2011 - 1 ठิ. L: 18.04.2014 - 1 sp., 21.04.2019 — 1 sp., near Quercus robur. 


\section{Infraorder EX O P O R I A Common, 1975}

\section{Superfamily H E P I A L O I D E A Stephens, 1829}

F a m i ly H E P I A L I D A E Stephens, 1829

Triodia sylvina (Linnaeus, [1760])

M a t e r i a l . D: 16.08.2007, 23.08.2007, 01.09.2007, 26.08.2011, 29.08.2015, 04.09.2015, 13.08.2016, 27.08.2016, 28.08.2020, 29.08.2020 - 1-5 sp. per night (at dusk).

Korscheltellus lupulina (Linnaeus, 1758)

M a t e r i a l. D: $23.05 .2015-1$ sp., 13.05.2016 - 1 sp., 25.05.2018 - 1 sp., $18.05 .2019-1$ sp. C: $14.05 .2014-1$ sp. L: 27.05.2000 - 1 sp., 25.05.2005 - 2 sp., 30.05.2005 - 1 sp., 30.05.2006 - 2 sp., 01.06.2006 - 1 sp., 15.05.2007 — 1 sp., 16.05 .2007 40 sp. in dusk, 26.05 .2007 - 1 sp., 31.05.2007 - 1 sp., 04.06.2007 — 1 sp.

\section{Inf r a o r der HET E R O N E U R A Tillyard, 1918}

\section{S u p e r f m i ly NEPT I C U L O I D E A Stainon, 1854}

\section{Family NEPT IC ULIDAE Stainton, 1854}

* Stigmella prunetorum (Stainton, 1855) (Figs. 1-2)

M a t e ri a l . D: 21.10.2018 - 1 mine on Prunus cerasus, 28.10.2018 - 1 mine on Prunus cerasus and 2 mines on Prunus domestica, 03.11.2018 - 1 mine on Prunus cerasus, 26.10.2019 - 6 mines on Prunus cerasus, 02.11.2019 - 2 mines on Prunus cerasus, 31.10.2020 — 4 mines on Prunus domestica, all empty.

\section{* Stigmella aceris (Frey, 1857) (Fig. 3)}

M a t e r i a l . L L and P: 03.06.2018 - 9 mines on Acer campestre, 06.06.2019 - 3 mines on Acer campestre, 22.06.2019 - tens of mines on Acer campestre and Acer tataricum, 02.08.2020 - 10 mines on Acer tataricum, 09.08.2020 — tens of mines on Acer tataricum, 23.08.2020 — tens of mines on Acer campestre, all empty.

\section{* Stigmella tiliae (Frey, 1856) (Figs. 4-5)}

M a t e r i a l . L: 03.07.2020 - 2 empty mines on Tilia cordata, 18.07.2020 - 2 empty mines at the same place.

* Stigmella salicis (Stainton, 1854) (Fig. 6)

M a t e ri a l. P: $18.10 .2020-1$ empty mine on Salix cinerea.

\section{* Stigmella trima cule lla (Haworth, 1828) (Fig. 7)}

M a t e $\mathbf{r}$ i a l . P: 08.07.2018 - 1 mine with a dead larva and 4 empty mines, 02.09.2018 - 1 empty mine, 26.06.2019 - 2 mines with larvae and 2 empty mines, 06.07.2019 - 1 empty mine, 13.07.2019 - 4 empty mines, 06.10.2019 - 1 mine with a dead larva and 3 empty mines, 26.06.2020 — 3 empty mines, all on Populus nigra.

* Stigme lla as s imile lla (Zeller, 1848) (Fig. 8) Populus alba.

M a t e ri a l . P: 07.07.2019 — 4 empty mines and 2 mines with dead larvae on Populus tremula, 19.07.2020 — 1 empty mine on

* Stigmella plagicole lla (Stainton, 1854) (Fig. 9)

M a t e r i a l . D: 21.10.2018 - 9 mines on Prunus cerasus, 28.10.2018 - 14 mines on Prunus cerasus and 10 mines on Prunus domestica, 03.11.2018 - 11 mines on Prunus cerasus, 07.09.2019 - 3 mines on Prunus domestica, 28.09 .2019 - 11 mines on Prunus cerasus, 13.10.2019 - 3 mines on Prunus cerasus, 19.10.2019 - 4 mines on Prunus cerasus, 26.10.2019 - 12 mines on Prunus cerasus, 19.09.2020 - 3 mines on Prunus domestica and 8 mines on Prunus cerasus, 31.10.2020 - 1 mine on Prunus armeniaca and 1 on Prunus domestica, all empty. C: 29.05.2019 - 1 mine with a larva on Prunus armeniaca.

* Stigme lla vis cere lla (Stainton, 1853) (Figs. 10-11)

M a t e r i a l . L: 22.06.2019 - 1 empty mine on Ulmus, 07.07.2019 - 30 empty mines on Ulmus.

* Stigm ella le m n is c ella (Zeller, 1839) (Figs. 12-13)

M a t e r i a l. L: 03.06.2018 - 2 empty mines, 04.07.2019 - 2 mines with dead larvae and 18 empty mines, all on Ulmus. P: 09.06.2018 - 1 empty mine, 07.07.2019 - 1 empty mine, all on Ulmus.

\section{* Stigmella basiguttella (Heinemann, 1862) (Fig. 14)}

M a t e r i a l . P: 08.07.2018 - 1 empty mine, 14.10.2018 - 1 mine with a dead larva and 2 empty mines, 27.10.2018 — 4 empty mines, 07.07.2019 - 1 empty mine, 13.07.2019 - 2 empty mines, 28.07.2019 — 2 empty mines, 06.10.2019 - 2 empty mines and 2 mines with dead larvae, 14.10.2019 - 1 mine with a dead larva, all on Quercus robur.

\section{* E ta in ia Io u is ella (Sircom, 1849) (Figs. 15-16)}

M a t e r i a l. L: 07.06.2020 - 7 mines on seeds of Acer campestre. 


\section{* Ectoedemia hannoverella (Glitz, 1872)}

M a t e r i a l. D: 27.05.2016 — 1 sp. P: 06.10.2019 — 1 mine, 14.10.2019 — 1 mine, 11.10 .2020 - 8 mines, all in fallen leaves of Populus nigra.

* E c to ede mia argyropeza (Zeller, 1839) (Figs. 17-18) half an hour).

M a t e ri a l . P: 06.10.2019 - thousands of mines in fallen leaves of Populus tremula (more than 120 mines were observed in a

Family OPOSTEG I A E Meyrick, 1893

* Opostega spatulella Herrich-Schäffer, [1855]

M a t e r i a l. D: $18.05 .2019-1$ sp., 01.05.2020 - 2 sp.

Ps e udopostega a urite lla (Hübner, [1813])

M a t e ri a l . D: 12.06.2015, 19.06.2015, 26.06.2015, 27.05.2016, 24.06.2016, 27.06.2017, 09.06.2018, 31.05.2019, 21.06.2019, 12.06.2020 - 1 sp. per night. L: 31.05.2007 — 1 sp. at dusk.

* P seudopostega crepusculella (Zeller, 1839)

M a t e ri a l. D: $12.06 .2015-1$ sp.

Superfamily A D E O I D A Bruand, 1850

Family ADELIDAE Bruand, 1850

Nemophora degeerella (Linnaeus, 1758) s. l.

M a t e ri a l. $\quad$ D: $23.06 .2017-2$ + $q, 27.06 .2017-1$ q, 13.06.2020- 1 q. L: 11.06.2001, 12.06.2001, 21.06.2001, 01.06.2006, 04.06.2006, 06.06.2006, 12.06.2006, 26.05.2007, 29.05.2007, 04.06.2007, 06.06.2007, 22.05.2008, 31.05.2008, 29.05.2010, 07.06.2016, 03.06.2018, 06.06.2018, 06.06.2019 — 1-20 per day. P: 30.05.2020 — 1 sp., 07.06 .2020 — 1 sp.

* Nemophora fasciella (Fabricius, 1775)

M a teria l. L: $06.06 .2006-1$ sp.

Ade la reamurella (Linnaeus, 1758)

M a t e ri a l . L and P: 27.04.2000, 04.05.2000, 01.05.2001, 30.04.2002, 11.05.2003, 13.05.2003, 25.04.2004, 02.05.2004, 14.05.2006, 10.05.2009, 16.05.2009, 02.05.2010, 02.05.2011, 10.05.2011, 18.05.2011, 22.04.2012, 01.05.2014, 24.04.2016, 08.05.2017, 14.05.2017, 30.04.2018, 01.05.2018, 06.05.2018, 19.05.2019, 20.04.2020, 02.05.2020 - 1-50 per day.

* Cauchas fibule lla ([Denis et Schiffermüller], 1775)

M a t e ri a l. P: 11.05.2020 - tens of sp. at glades with Veronica chamaedrys.

* Cauchas rufimitrella (Scopoli, 1763)

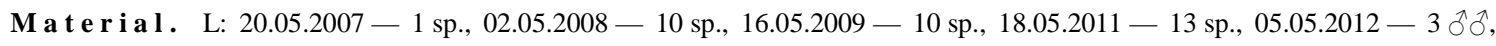
10.05.2014 — 1 sp., 18.05 .2014 - 1 sp., 14.05.2017 — 1 sp., 02.05 .2020 - 3 sp., mostly on Alliaria petiolata.

Nematopogon swammerdame lla (Linnaeus, 1758)

M a t e r i a l . L: 27.04.2001, 01.05.2001, 02.05.2002, 11.05.2003, 13.05.2003, 25.04.2004, 16.05.2004, 24.04.2005, 27.04.2005, 05.05.2005, 19.05.2005, 23.05.2005, 30.04.2007, 05.05.2007, 12.05.2007, 13.05.2007, 15.05.2007, 26.05.2007, 03.05.2009, 10.05.2009, 16.05.2009, 25.04.2010, 02.05.2010, 10.05.2010, 02.05.2011, 10.05.2011, 18.05.2011, 21.04.2012, 05.05.2012, 01.05.2014, 17.04.2016, 24.04.2016, 26.04.2017, 27.04.2017, 30.04.2018, 21.04.2019, 05.05.2019, 19.05.2019 - 1-20 per day.

F a mi ly I N C UR VAR I I A E Spuler, 1898

* Incurvaria pectinea Haworth, 1828

M a t e r i a l. P: 27.04.2018 - 5 sp. (1 ô coll.), 14.04.2019 - 3 sp. (1 ô coll.), birch forests.

Incurvaria mascule Ila ([Denis et Schiffermüller], 1775)

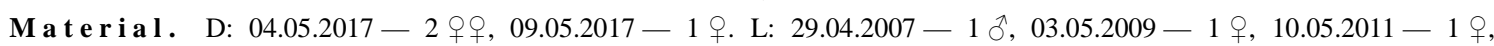
16.04.2017-1 1 +.

S u perfamily T ISCHERIOIDEA Spuler, 1898

F a mily T I S C H E R I I D A E Spuler, 1898

* Tis cheria e kebladella (Bjerkander, 1795) (Fig. 19)

M a t e r i a l . L L and P: 08.07.2018, 08.09.2018, 16.09.2018, 14.10.2018, 27.10.2018, 22.06.2019, 26.06.2019, 07.07.2019, 13.07.2019, 28.07.2019, 29.09.2019 — numerous mines, on Quercus robur. 
* Tischeria dodonaea Stainton, 1858 (Fig. 20)

M a t e r i a l . P: 16.09.2018 - 3 mines, 27.10.2018 - 1 mine, 26.06.2019 - 3 mines, on Quercus robur.

* Tis cheria de c idua Wocke, 1876 (Fig. 21)

M a t e r i a l . P: 08.07.2018, 19.07.2018, 28.07.2018, 08.09.2018, 16.09.2018, 14.10.2018, 27.10.2018, 26.06.2019, 07.07.2019, 13.07.2019, 28.07.2019 - 1-40 mines per day, on Quercus robur.

* Coptotriche angusticollella (Duponchel, [1843])

M a te ri a l. D: $31.08 .2018-1$ sp.

Superfamily T I NEO I D A Latreille, 1810

Family ERIOCOT TIDAE Spuler, 1898

Deuterotinea casanella (Eversmann, 1844)

M a t e r i a l . D: 02.10 .2015 - 1 sp., 03.10.2015 - 1 sp., 29.10 .2018 - 1 sp., $13.10 .2019-8$ sp., $16.10 .2020-6$ sp. C: 17.10.2009 - 2 sp., 24.10.2009 - 1 sp., 18.10.2013 - 1 sp., 25.10.2013 - 2 sp., 16.10 .2015 - 1 sp., 29.10 .2015 - 1 sp., 22.10 .2016 8 sp., 08.11.2016 — 5 sp., 27.10.2017 — 1 sp. L: 06.11.2016 — 1 sp., 20.10.2018 — 1 sp.

Family PS Y CH I D A E Boisduval, 1829

Ta leporia tubulosa (Retzius, 1783)

M a t e r i a l . L: 14.05.2017 — 1 larval case, ổ hatched on 03.06.2017. P: 11.05.2019 — tens of larval cases, 16.05.2019 — tens of larval cases, 08.06.2019-1 1 .

* Epichnopterix plumella kovacsi Sieder, 1955

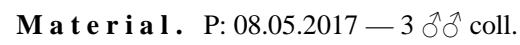

Bijugis bombyce lla ([Denis et Schifermüller], 1775)

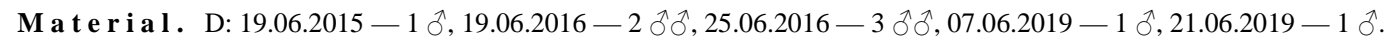

Canephora hirsuta (Poda, 1761)

M a t e r i a l . L and P: 03.06.2006, 13.05.2007, 04.06.2007, 28.07.2008, 18.05.2011, 05.06.2011 — single larval cases.

F a mily T I N E I D A L Latreille, 1810

* Montescardia tessulatellus (Zeller, 1846)

M a t e r i a l. P: 02.05.2017 — 1 sp., aspen-birch forest.

* Morophaga choragella ([Denis et Schiffermüller], 1775)

M a t e r i a l . D: 16.05.2014 - 1 sp., 27.05.2016 - 1 sp., 24.07.2019 - 1 sp., 07.06.2020 - 1 sp. C: 26.07 .2011 - 1 sp.

* Archinemapogon yildizae Koçak, 1981

M a t e r i a l. D: 23.05.2015 — 1 sp., 07.08.2015 — 1 sp., 09.05.2018 - 2 sp., 09.06.2018 - 1 sp.

Nemapogon picarella (Clerck, 1759)

M a t e r i a l . D: 27.05.2016 - 1 sp. P: 31.05.2019 - 1 sp., on trunks of Quercus robur.

* Nemapogon granella (Linnaeus, 1758)

M a t e ri a l. C: $02.05 .2010-1$ sp. indoors.

Nemapogon variatella (Clemens, 1860)

M a t e r i a l . D: 23.05 .2015 - 1 sp., 10.07 .2015 - 1 sp., 31.07.2015 - 1 sp., 13.05.2016 - 1 sp., 15.07.2016 - 1 sp., 26.07.2016 - 1 sp.

Neurothaumasia ankerella (Mann, 1867)

M a t e ri a l. D: 23.07.2008 - 1 sp., 24.05.2014 - 1 sp., 28.06.2015 - 1 sp., 27.06.2017 - 1 sp.

* Infurcitinea albicomella (Stainton, 1851)

M a te ri a l. D: $16.08 .2019-1$ sp. L: $22.06 .2019-1$ sp. P: $23.07 .2017-2$ sp., $09.06 .2018-2$ sp., $28.07 .2018-7$ sp., 07.07.2019 — 1 sp., on trunks of Quercus robur.

* Infurcitinea ignicomella (Heydenreich, 1851)

M a teria l. D: 03.06.2016 - 1 sp. 
S. O. DEMYANENKO, O. V. BIDZILYA, E. A. KAROLINSKIY New records of Lepidoptera (Insecta) of Severodonetsk (Luhansk Region, Ukraine) and its environs

Ateliotum hungaricellum (Zeller, 1839)

M a t e r i a l . D: 23.07.2008, 26.06.2015, 28.06.2015, 10.07.2015, 24.07.2015, 31.07.2015, 14.08.2015, 01.07.2016, 03.07.2016, 17.07.2016, 26.07.2016, 23.06.2017, 27.06.2017, 28.07.2017, 20.07.2018, 27.07.2018, 07.06.2019, 21.06.2019, 27.06.2019, 13.06.2020, 28.06.2020, 03.07.2020, 08.08.2020 - 1-3 sp. per night.

* Trichophaga scandinaviella Zagulajev, 1960

M a t e ri a l. D: $03.06 .2016-1$ sp.

* Tineola bis selliella (Hummel, 1823)

M a t e r i a l. D: 03.07.2015 - 1 sp., 07.06.2020 - 1 sp., 26.06.2020 - 1 sp. C: 2000-2002 - 1 ô.

* Tinea pellionella Linnaeus, 1758

M a t e r i a l . C: 08.07.2011, 07.07.2012, 02.08.2013, 29.06.2014, 18.09.2014 — single sp. indoors.

Tinea omichlopis Meyrick, 1928

M a t e ri a l. D: $13.05 .2016-1$ sp., 27.05.2016 - 1 sp. C: $2000-2002-1$ sp.

Tinea trinotella Thunberg, 1794

M a te ri a l. D: $04.05 .2018-1$ sp.

Niditinea fuscella (Linnaeus, 1758)

Material. D: $10.05 .2014-1$ ㅇ.

* Monopis la evigella ([Denis et Schiffermüller], 1775)

M a t e ri a l. D: 31.07.2015 - 1 sp., 01.05.2020 - 1 sp.

* Monopis obvi ella ([Denis et Schiffermüller], 1775)

M a t e r i a l . D: 05.06.2016, 19.06.2016, 03.07.2016, 23.06.2017, 27.06.2017, 17.08.2018, 31.08.2018, 08.09.2018, 26.06.2020, 28.06.2020 - 1 sp. per night.

* Monopis im ella (Hübner, [1813])

M a teria l. L: $10.05 .2016-1$ sp.

* Monopis pallidella Zagulajev, 1955

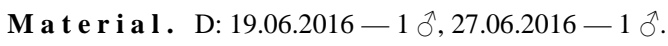

Monopis monachella (Hübner, 1796)

M a t e ri a l . D: 07.08.2013， 30.05.2015，28.06.2015，24.07.2015，27.05.2016，18.05.2019，24.05.2019， 09.08.2019, 28.09.2019 - 1-2 sp. per night. L: 16.05 .2007 - 2 sp. at dusk.

Wegneria panchalcella (Staudinder, 1871)

M a t e ri a l. D: $07.08 .2015-1$ sp., 19.08.2017 - 1 sp.

S u p e r f a m i l y G R A C I L L A R I O I D E A Stainton, 1854

F a mily B UC C U L A T R I C I D A E Fracker, 1915

* B и с сulatrix noltei Petry, 1912 (Fig. 22)

M a t e r i a l . L and P: 06.06.2019, 08.06.2019, 22.06.2019 — secondary mines on Artemisia vulgaris.

* B u c culatrix albedinella (Zeller, 1839) (Fig. 23)

M a t e r i a l. L: 04.07.2019 — 5 empty mines, 15.09.2019 — 2 empty mines, on Ulmus.

* Bucculatrix frangutella (Goeze, 1783)

M a t e r i a l. D: $25.07 .2015-1$ sp., 31.07.2015 - 1 sp., 27.07.2018 - 2 sp., $31.07 .2020-1$ sp. P: $16.09 .2018-2$ empty mines, 06.10.2019 — 10 mines, all on Frangula alnus.

* B u c culatrix bech ste in ella (Bechstein et Scharfenberg, 1805)

M a t e r i a l. D: 10.07.2015 - 1 sp. P: 08.06.2018 - mines on Pyrus, 09.06.2018 - mines on Pyrus, 08.07.2018 — mines on Crataegus, 05.08.2018 — 1 sp., 08.09.2018 — mines on Malus, 11.05.2019 - 1 sp. on a trunk of Pyrus.

* B u c c u latrix c idare lla (Zeller, 1839) (Figs. 24-25)

M a t e ri a l. P: 07.07.2019 - 16 mines on Alnus glutinosa.

* B u c culatrix u Ime lla Zeller, 1848

M a t e r i a l. $\quad$ P: 23.07 .2017 - 1 sp., 03.09.2017 - 1 sp., 08.07 .2018 - 3 sp., 14.07.2018 - 1 sp., 12.08.2018 - 1 sp., 08.09.2018 - 2 cocoons, 16.09.2018 - 1 cocoon, 11.05.2019 — 15 sp., 31.05.2019 — 10 sp., all on trunks of Quercus robur; 06.10.2019 2 mines, 14.10.2019 — 4 mines, all on Quercus robur. 


\section{F a mi l y G R A C I L L A R I I D A E Stainton, 1854}

* Micrurapteryx kollariella (Zeller, 1839) (Figs. 26-27)

M a t e r i a l. P: $26.07 .2020-1$ empty mine and 1 cocoon with a pupa, $01.08 .2020-1$ empty mine and 1 empty cocoon, all on Chamaecytisus.

* Caloptilia fidella (Reutti, 1853)

M a teria l. D: $31.07 .2015-1$ \%.

* Caloptilia he midactyle lla ([Denis et Schiffermüller], 1775)

M a t e r i a l. D: 10.04.2010 - 1 sp., indoors in the daytime, 01.11.2011 - 1 dead sp., 2012 - 1 sp. L: 06.04 .2008 - 1 sp.

* Caloptilia semifascia (Haworth, 1828)

M a teria l. L: $07.11 .2010-1 \mathrm{sp}$.

* Caloptilia stigmatella (Fabricius, 1781)

M a t e r i a l. D: $27.06 .2017-1$ sp., $16.10 .2020-1$ sp.

* Gracillaria loriolella Frey, 1881

M a te ri a l. D: 01.05.2020-1 sp.

* Aspilapteryx tringipennella (Zeller, 1839)

M a t e ri a l. D: $24.05 .2014-1 \mathrm{sp.}$

* Calybites phasianipennella (Hübner, [1813])

M a t e ri a l . D: 24.07.2015 - 2 sp., 01.07.2016 - 1 sp., 26.07.2016 - 1 sp., 31.07.2016 - 1 sp., 31.05.2019 - 1 sp., 09.08.2019 - 1 sp.

* Calybites quadrisignella (Zeller, 1839)

M a te ri a l. D: $10.07 .2015-1$ sp., 09.08.2019 - 1 sp.

* Acrocercops brongniardella (Fabricius, 1798)

M a teria l. L: $28.10 .2018-3$ sp. under bark of Quercus robur. P: $27.05 .2018-2$ mines, $10.06 .2018-3$ mines, 14.10.2018 — 1 mine, 16.05.2019 — 1 mine, 13.07.2019 — 6 mines, all on Quercus robur.

* Le ucospilapteryx om is sella (Stainton, 1848) M a t e ri a l. D: 09.08.2019-1 sp.

* Ornixola caudulatella (Zeller, 1839)

M a te ri a l. D: $26.06 .2020-1 \mathrm{sp.}$

* Callisto denticulella (Thunberg, 1794)

M a t e r i a l. D: 30.04 .2018 - 1 sp., in the daytime on Malus.

* Parornix anguliferella (Zeller, 1847)

M a te ri a l. D: $24.06 .2016-1 \mathrm{sp.}$

Parornix petiolella (Frey, 1863)

M a t e ri a l . D: 26.06.2015 - 1 sp., 24.07.2015 - 1 sp., 21.10.2019 - 1 mine on Malus. P: 13.07.2019 - 6 mines, 28.07.2019 - 12 mines on Malus.

* Phyllonorycter abrasella (Duponchel, [1843])

M a t e r i a l . P: 11.05.2019 - 1 sp., 07.07.2019 - 1 sp., 11.05.2020 - 1 sp., all on trunks of Quercus robur.

* Phyllonorycter harrisella (Linnaeus, [1760])

M a t e r i a l. P: 11.05.2019-1 sp. on a trunk of Quercus robur.

* Phyllonorycter roboris (Zeller, 1839)

M a t e r i a l . D: 27.06 .2017 - 1 sp. L: 25.04.2008 - 3 sp., 21.04.2013 - 1 sp., 19.04 .2014 - tens of sp., $26.04 .2017-1$ sp., 27.04.2017 - 1 sp., mainly on trunks of Quercus robur. P: 25.06.2017 - 7 sp., $06.05 .2018-1$ sp., $28.07 .2018-1$ sp., $05.08 .2018-$ 5 sp., 11.05.2019 - 5 sp., 13.07.2019 - 1 mine (imago hatched on 23.07.2019), 30.05.2020 - 1 sp., $11.05 .2020-1$ sp., $31.05 .2020-$ 1 sp., all on trunks of Quercus robur.

\section{* Phyllonorycter quercifoliella (Zeller, 1839)}

M a t e ri a l. L: 06.05 .2007 - 1 sp., 21.04.2013 - 2 sp., 19.04.2014 - 50 sp., mainly on trunks of Quercus robur. P: 25.06.2017 - 1 sp., 27.05.2018 - 1 mine (imago hatched on 03.06.2018), 28.07.2018, 11.05.2019 - 2 sp., 11.05.2020 - 1 sp., all on trunks of Quercus robur. 


\section{* Phyllonorycter is siki (Kumata, 1963)}

M a t e r i a l. D: 06.09 .2019 - 1 sp. L: 03.07.2020 - 15 mines on Tilia cordata, 03.07.2020 — tens of mines on Tilia cordata. P: 10.06.2020 - 1 mine on Tilia cordata.

* Phyllonorycter rajella (Linnaeus, 1758)

M a t e ri a l. P: 27.04.2019-1 ठ, alder-birch forest.

\section{* Phyllonorycter froliechiella (Zeller, 1839)}

M a t e ri a l. P: 04.05.2019 - 2 sp. on trunks of Alnus glutinosa, 26.06.2019 - 1 mine (with a pupa, imago hatched 02.07.2019), 07.07.2019 - 1 sp. on Ulmus, 13.07.2019 - 1 mine (with a pupa, imago hatched on 23.07.2019) on Alnus glutinosa, 28.07.2019 - 18 mines (with pupae, 3 sp. of imago hatched on 31.07.2018, 1 sp. of imago hatched on 02.08.2019, 4 sp. of imago hatched on 04.08.2019, 2 sp. of imago hatched on 06.08.2019, 2 sp. of imago hatched in 2020) on Alnus glutinosa, 06.10.2019 - 10 mines with larvae on Alnus glutinosa, 30.05.2020 — tens of sp., birch-alder forest, 31.05.2020 - 1 sp.

* Phyllonorycter schreberella (Fabricius, 1781) (Figs. 28-30)

M a t e r i a l. L: 15.09.2019 - 1 mine with a cocoon on Ulmus.

* Phyllonorycter agile lla (Zeller, 1846)

M a t e r i a l . L: 01.03.2014 - 3 sp., 02.03.2014 - 3 pair, 22.03.2014 - 2 sp., 03.03.2016 - 1 sp., 09.04.2016 - 1 sp., 12.11.2017 - 1 sp., 28.10.2018 - 20 sp., 31.03.2019 - 2 sp., 15.09.2019 - 1 sp., 29.03.2020 - 10 sp., under bark of Quercus robur, Populus and other trees. P: 14.04.2019 — 1 sp. under bark of Betula.

* Phyllonorycter cerasicolella (Herrich-Schäffer, [1855])

M a terial. D: $27.06 .2017-1$ 万.

* Phyllonorycter apparella (Herrich-Schäffer, [1855])

M a t e ri a l . D: 07.08.2015 - 1 sp. L: 01.03.2014 - 1 died ô under bark. P: 09.08.2015 - 2 sp. on a trunk of Quercus robur, near Populus alba/canescens.

\section{* Phyllonorycter comparella (Duponchel, [1843])}

M a t e r i a l. D: 28.09.2014 - 1 sp. in the daytime. P: 07.07.2019 - 6 mines, 04.08.2019 - 4 mines, $18.08 .2019-15$ mines, 19.07.2020 — 2 mines, 23.08.2020 — 25 mines (2 sp. of imago hatched before 06.09.2020). All mines on Populus alba.

* Phyllonorycter sagitella (Bjerkander, 1790)

M a t e r i a l. P: 21.06.2020 - 2 mines with pupae (1 sp. of imago hatched the same day) on Populus tremula.

* Phyllonorycter acerifoliella (Zeller, 1839)

M a t e r i a l. L: 21.04.2013 - 3 sp., 19.04.2014 - 3 sp., 26.04.2017 - 1 sp.

* Phyllonorycter joannis i (Le Marchand, 1936) (Figs. 31-32)

M a t e r i a l. P: 27.06.2019 - 1 mine on Acer platanoides.

\section{* Cameraria ohride lla Deschka et Dimić, 1986}

M a t e r i a l. D: 15.09 .2012 - 1 sp., $17.08 .2018-1$ sp., $03.08 .2019-1$ sp., $09.08 .2019-1$ sp., $16.08 .2019-1$ sp., all in area without Aesculus hippocastanum. C: $09.09 .2013-1$ sp., 23.08.2014 - 1 sp., 30.07.2019 - 2 sp. daytime, 02.08.2019 - 1 sp. in the daytime, 28.08.2019 - $1 \mathrm{sp}$. in the daytime, all near Aesculus hippocastanum. P: 05.08.2018 - 1 sp. on trunks of Quercus robur, in an area without Aesculus hippocastanum.

* Phyllocnistis unipunctella (Stephens, 1834)

M a t e ri a l. D: $28.06 .2015-1$ sp., 03.07.2015 - 1 sp., 10.07.2015 - 1 sp., 24.07.2015 - 4 sp., $14.08 .2015-1$ sp., 25.06.2016 - 1 sp., 26.07.2016 - 1 sp., 17.08.2018 - 1 sp., 27.06.2019 - 1 sp. C: 19.06 .2018 - 1 sp. L: 28.10 .2018 - 1 sp. under bark of Quercus robur. P: 08.07.2018 - 1 empty mine, 08.06.2019 - 4 mines with larvae, 26.06.2019 - 3 mines, 06.07.2019 - 10 mines, 13.07.2019 — 3 mines, 04.08.2019 — 3 mines, all on Populus nigra.

\section{S u perfamily Y P O N M E U T O I D E A Stephens, 1829}

F a mily Y P O N O M E T I D A E Stephens, 1829

Yponomeuta evonymella (Linnaeus, 1758)

M a t e r i a l. D: 10.07.2015, 20.07.2018, 27.07.2018, 05.07.2019, 20.07.2019, 24.07.2020, 31.07.2020 — 1 sp. per night.

Yponomeuta cagnagella (Hübner, [1813])

M a t e r i a l . L: 17.05.2019, 19.05.2019 and 10.05.2020 — tens of larvae on Euonymus, 10.07.2020 — 1 sp.

Yponomeuta plumbella ([Denis et Schiffermüller], 1775)

M a t e r i a l. D: 03.07.2015 - 1 sp., 10.07.2015 - 3 sp., 02.10.2015 - 1 sp., 27.06.2017 — 1 sp. L: 01.07.2006, 04.07.2006, 18.06.2007, 30.06.2007, 19.07.2008, 03.07.2020, 10.07.2020 — 1-10 sp. per day. 
Ypo nomeuta sedella (Treitschke, 1832)

M a t e ri a l. D: $24.07 .2015-1$ sp.

* Swammerdamia pyrella (de Villers, 1789)

M a t e r i a l . D: 07.08.2013, 03.07.2015, 01.07.2016, 06.08.2016, 13.08.2016, 23.06.2017, 27.06.2017, 04.05.2018, 09.08.2019, 01.05.2020, 14.08.2020, 21.08.2020, 28.08.2020 - 1-2 sp. per night.

* Paraswammerdamia albicapitella (Scharfenberg, 1805)

M a t e r i a l . D: 10.07.2015 - 1 sp., 04.09.2015 - 1 sp., 31.05.2019 - 1 sp.

* Cedestis gysseleniella (Zeller, 1839)

M a t e ri a l. D: 03.06.2016 - 2 sp., 05.06.2016 - 1 sp.

Family ARG YRESTHIIDAE Bruand, 1850

* Argyresthia bonnetella (Linnaeus, 1758)

M a teri a l. L: 21.07.2016 - 1 sp.

\section{F a mily PLUT E L L I A E Guenée, 1845}

\section{Plutella xylostella (Linnaeus, 1758)}

M a t e r i a l . D: $12.06 .2015,19.06 .2015,26.06 .2015,28.06 .2015,03.07 .2015,10.07 .2015,24.07 .2015,31.07 .2015,07.08 .2015$, 04.09.2015, 18.09.2015, 25.09.2015, 02.10.2015, 08.04.2016, 02.05.2016, 13.05.2016, 24.06.2016, 01.07.2016, 06.08.2016, 19.08.2016, 23.06.2017, 27.06.2017, 28.07.2017, 04.05.2018, 09.05.2018, 25.05.2018, 02.06.2018, 06.07.2018, 20.07.2018, 27.07.2018, 17.08.2018, 31.08.2018, 08.09.2018, 18.05.2019, 07.06.2019, 21.06.2019, 05.07.2019, 20.07.2019, 03.08.2019, 16.08.2019, 06.09.2019, 14.09.2019, 01.05.2020, 07.06.2020, 12.06.2020, 19.06.2020, 26.06.2020, 03.07.2020, 24.07.2020, 08.08.2020, 14.08.2020, 28.08.2020, 11.09.2020, 16.10.2020 - 1-15 sp. per night; 18.05.2019 - numerous, in the daytime on Sinapis. L and P: 03.07.2002, 30.03.2004, 24.04.2005, 04.08.2006, 24.05.2007, 31.05.2007, 21.05.2008, 30.05.2009, 10.05.2010, 22.05.2010, 21.09.2012, 02.04.2013, 17.04.2014, 26.04.2014, 01.05.2014, 13.04.2016, 17.04.2016, 24.04.2016, 10.05.2016, 11.05.2016, 09.05.2016, 02.05.2017, 01.06.2017, 01.04.2018, 27.04.2019, 04.05.2019, 11.05.2019, 16.05.2019, 06.06.2019, 08.09.2019, 16.05.2020, 30.05.2020, 31.05.2020 — 1-100 sp. per day.

* Plutella porrectella (Linnaeus, 1758)

M a t e ri a l. D: $30.05 .2015-1$ sp., 27.05.2016 - 1 sp.

E idophas ia messingi ella (Fischer von Röslerstamm, 1839)

M a t e r i a l. $\quad$ D: $04.06 .2012-2$ sp., 03.06.2016 - 1 sp., 05.06.2016 - 2 sp., 19.06.2016 - 2 sp., $23.06 .2017-1$ sp., 27.06.2017 — 1 sp., 09.06.2018 - 2 sp., 31.05.2019 - 1 sp.

\section{F a mily G LY P H I P T E R I G I D A E Stainton, 1854}

* Glyphipterix forsterella (Fabricius, 1781)

M a t e ri a l. L: 27.05 .2017 - 1 sp., $10.05 .2020-1$ sp.

\section{F a mily Y P S O L O PH I A E Guenée, 1845}

* Ypsolopha mucronella (Scopoli, 1763)

M a teria l. L: $29.03 .2020-1$ sp.

* Yps o lopha horride lla (Treitschke, 1835)

M a t e r i a l . D: 25.09.2015 - 1 sp. P: 08.07.2018 - 1 sp. on trunks of Pyrus, 19.07.2018 - 1 sp. at the same place.

* Yps olopha lucella (Fabricius, 1775)

M a t e ri a l. D: $19.06 .2020-1$ sp. P: 01.09.2002 — 1 sp., 08.06.2019 - 1 sp.

* Yps o lopha a lp e I la ([Denis et Schiffermüller], 1775)

M a t e ri a l . D: 29.08 .2015 - 1 sp. P: 25.06 .2017 - 1 sp. near Quercus robur, 23.07.2017 - 1 sp. at the same place, 08.09.2018 - 1 sp. on trunks of Pyrus.

* Ypsolopha us te lla (Clerck, 1759)

M a t e r i a l. L: 02.03.2014 - 1 sp. under bark of a tree.

* Ypsolopha sequella (Clerck, 1759)

M a t e ri a l. L: $22.06 .2019-1$ sp. on trunks of a tree. 
S. O. DEMYANENKO, O. V. BIDZILYA, E. A. KAROLINSKIY New records of Lepidoptera (Insecta) of Severodonetsk (Luhansk Region, Ukraine) and its environs

Yps olopha vittella (Linnaeus, 1758)

M a t e r i a l . L: L: 04.07.2006 — 1 sp., 17.06.2007 — 1 sp., on trunks of Quercus robur. P: 01.06.2014 — 1 sp., $22.06 .2014-$ 1 sp., on trunks of Quercus robur.

Ypsolopha chazariella (Mann, 1866)

M a t e r i a l. D: 27.06.2016 - 1 sp., 06.07.2018 - 1 sp., 28.06.2020 - 1 sp.

* Och s enhe imeria vac cule lla Fischer von Röslerstamm, 1842 same place.

M a t e r i a l . P: $02.09 .2018-30$ sp. under bark of Betula, 08.09.2018 - 10 sp. at the same place, $27.10 .2018-1$ sp. at the

F a mily B E D E L I I D A E Meyrick, 1880

* Bedellia somnulentella (Zeller, 1847)

M a t e r i a l . D: 24.07.2015, 14.08.2015, 04.09.2015, 12.09.2015, 25.09.2015, 03.06.2016, 27.06.2016, 15.07.2016, 28.07.2017, 19.08.2017, 27.07.2018, 17.08.2018, 31.08.2018, 08.09.2018, 21.06.2019, 03.08.2019, 09.08.2019, 16.08.2019, 06.09.2019, 28.09.2019, 13.06.2020, 29.08.2020 - 1-3 sp. per night.

F a mily LY O N E T I D A E Stainton, 1854

* Leucoptera malifoliella (O. Costa, [1836]) (Fig. 33)

M a t e ri a l. D: 06.07.2019 - 1 mine on Malus, 19.09.2020 - single mines on Prunus cerasus and Malus. P: 28.07.2018 1 mine on Pyrus, 12.08.2018 — tens of mines on Malus, 13.07.2019 — tens of mines on Malus.

* Lyonetia prunifoliella (Hübner, 1796)

M a te ri a l. D: $28.06 .2015-1$ sp.

S up e r f a mily G E L E C H I O I D E A Stainton, 1854

Family A U T OS T I H I A E Le Marchand, 1947

O ego c o nia de a uratella (Herrich-Schäffer, 1854)

M a t e r i a l. D: 28.06.2015 — 1 sp., 03.07.2015 — 1 sp., 10.07.2015 — 1 sp., 24.06.2016 — 1 sp., 05.07.2019 — 1 sp. C: $2002-$ $2002-2$ ôे. P: 08.07.2018 - 1 sp., on trunks of Quercus robur.

F a mily B L A S T O B A S I D A E Meyrick, 1894

B lastobas is phycidella (Zeller, 1839)

M a t e r i a l . D: 14.08.2013, 24.05.2014, 30.05.2015, 19.06.2015, 27.05.2016, 03.06.2016, 05.06.2016, 19.08.2016, 23.06.2017, 25.05.2018, 02.06.2018, 09.06.2018, 17.08.2018, 18.05.2019, 24.05.2019, 31.05.2019, 03.08.2019, 12.06.2020, 13.06.2020 - 1-2 sp. per night. P: 11.06 .2016 - 1 q, 27.05.2018 - 1 sp., 09.06.2018 - 3 sp., 08.06.2019 - 1 sp., mostly on trunks of Quercus robur and Pyrus.

F amily OEC OPHORIDAE Bruand, 1850

Promalact is procerella ([Denis et Schiffermüller], 1775)

M a t e ri a l. D: 27.06.2017 - 1 sp., 28.06.2020-1 sp.

* Schiffermuelleria schaefferella (Linnaeus, 1758)

M a t e r i a l. D: $23.05 .2015-1$ sp., $07.05 .2017-1$ sp. at dusk, $30.04 .2018-1$ sp. in the daytime, $08.05 .2018-1 \mathrm{sp}$. in the daytime, $09.05 .2018-1 \mathrm{sp}$.

* Denis ia simile lla (Hübner, 1796)

M a t e r i a l. P: $28.05 .2018-1$ sp. on trunks of Pyrus, 09.06.2018 - 2 sp. at the same place.

Decantha borkhausenii (Zeller, 1839)

M a t e ri a l. P: 25.06.2017 - 1 sp. on trunks of Quercus robur.

Meta lampra cinnamomea (Zeller, 1839)

M a t e r i a l. D: 19.06 .2016 - 1 sp., 25.06.2016 - 2 sp., 27.06.2017 - 2 sp., 21.06.2019 - 1 sp. P: $12.06 .2016-1$ sp., 25.06.2017 — 2 sp., 09.06.2018 - 5 sp., 08.07.2018 - 3 sp., 14.07.2018 - 1 sp., 08.06.2019 — 12 sp., on trunks of Quercus robur.

* Borkhausenia fuscescens (Haworth, 1828)

M a t e ri a l. D: 06.08.2016 - 1 sp., 24.07.2020 - 1 sp. 


\section{Crassa un it e lla (Hübner, 1796)}

M a t e r i a l . D: 19.06.2015, 10.07.2015, 19.06.2016, 24.06.2016, 25.06.2016, 27.06.2016, 23.06.2017, 27.06.2017, 06.07.2018, 27.07.2018, 21.06.2019, 27.06.2019, 26.06.2020 — 1-8 sp. per night. P: 25.06.2017 — 2 sp., 08.07.2018 — 7 sp., 08.06 .2019 - 1 sp., on trunks of Quercus robur.

E p i c a l l i m a form o s e l l a ([Denis et Schiffermüller], 1775)

M a t e r i a l . D: 19.06.2015, 19.06.2016, 01.07.2016, 23.08.2016, 23.06.2017, 27.06.2017, 21.06.2019-1 sp. per night. P: 25.06.2017 — $1 \mathrm{sp.} \mathrm{on} \mathrm{trunks} \mathrm{of} \mathrm{Quercus} \mathrm{robur.}$

P l e urota pyrope I I a ([Denis et Schiffermüller], 1775)

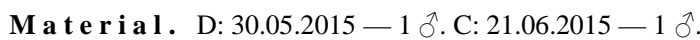

F a mily L Y P U S I D A E Herrich-Schäffer, 1857

* Lypusa maure l la ([Denis et Schiffermüller], 1775)

M a t e ri a l. L: $14.05 .2017-1 \mathrm{sp.}$

F a mily C H I M A B A C H I D A E Heinemann, 1870

* Di urnea fage lla ([Denis et Schiffermüller], 1775)

M a t e r i a l . D: 14.04.2013 - 1 sp., 18.04.2014 - 1 sp., 08.04.2016 - 4 sp. C: 07.04 .2009 - 1 sp. L: 29.03 .2007 - 2 sp., 19.04.2014 - 2 sp., 09.04.2016 - 1 sp., 10.04.2016 - 1 sp., 13.04.2017 - 3 sp., 27.04.2017 - 2 sp., on trunks of trees.

* D i urne a lips i e ll a ([Denis et Schiffermüller], 1775)

M a t e r i a l . L: 14.10 .2015 - 1 sp., 18.10.2015 - 5 sp. P: 27.10.2012 - 1 sp., 15.10.2017 - 1 sp., 04.11.2017 - 2 sp., 15.10.2018 - 1 sp., 20.10.2018 - 10 sp., 27.10.2018 - 3 sp., $14.10 .2019-6$ sp., birch and oak forests.

* Dasystoma salic ella (Hübner, 1796)

M a t e ri a l. L: 03.04.2016 - 1 sp.

Family ELACH IS T I A E Bruand, 1850

E I a ch is ta dum os a Parenti, 1981

M a t e ri a l. D: $10.05 .2014-1 \mathrm{sp}$.

* E Iachista littoricola Le Marchand, 1938

M a teria l. D: $04 \cdot 09.2015-1 \mathrm{sp}$.

* E Iachista anserinella Zeller, 1839

M a t e ri a l. D: $11.05 .2016-1 \delta$.

* E Iachista maculicerusella (Bruand, 1859)

M a t e r i a l. D: 24.05.2016 — 1 sp., 25.06.2016 — 2 sp., 28.07.2017 — 1 sp.

* B is el a chista utone lla Frey, 1856

M a t e r i a l. D: 27.06.2017 - 2 sp.

B Iastodacna atra (Haworth, 1828)

M a t e r i a l. D: 14.08.2013, 30.05.2015, 03.07.2015, 10.07.2015, 19.08.2017, 16.08.2019, 08.08.2020 — 1 sp. per night.

* Dystebenna stephensi (Stainton, 1849)

M a t e ri a l . $\quad$ P: 25.06 .2017 - 3 sp., 09.06.2018 - 4 sp., 10.06.2018 - 1 sp., $08.06 .2019-2$ sp., 21.06 .2020 - 1 sp., 03.07.2020 - 4 sp., on trunks of Quercus robur.

Family DEPRESSAR I I A E Meyrick, 1883

* S emioscopis a vellanella (Hübner, 1793)

M a teria l. L: $24.03 .2007-1$ sp.

S e mi s s copis ste inkell neriana ([Denis et Schiffermüller], 1775)

M a t e ri a l. L: $16.04 .2012-1 \mathrm{sp.}$

* Agonopterix arenella ([Denis et Schiffermüller], 1775)

M a t e ri a l. $\quad$ D: $18.04 .2014-1$ sp., 09.05 .2018 - 1 sp., 25.05.2018 - 1 sp. L: 27.08.2003, 11.03.2007, 05.05.2007, 15.05.2007, 12.04.2008, 22.05.2008 — 1 sp. per day, mainly under bark of trees. 
* Agonopterix propinquella (Treitschke, 1835)

M a t e r i a l. D: 26.06.2015, 27.06.2016, 03.07.2016, 15.07.2016, 23.08.2016, 03.07.2020, 07.10.2020 — 1 sp. per night.

Agonopterix alstromeriana (Clerck, 1759)

M a t e r i a l . D: $03.04 .2010-1$ sp., 30.10.2010 - 1 sp. indoors, 08.04.2016 — 2 sp. L: $22.03 .2014-1$ sp. under bark of trees. P: $12.04 .2017-1 \mathrm{sp}$.

* Agonopterix heracliana (Linnaeus, 1758)

M a t e ri a l. L: 11.03.2007 - 3 sp. under bark of trees.

* Depressaria depressana (Fabricius, 1775)

M a t e r i a l. D: 15.07.2016 — 3 sp., 17.07.2016 — 1 sp., 13.08.2016 — 1 sp., 21.07.2017 — 1 sp., 28.07.2017 — 1 sp.

* Depressaria emeritella Stainton, 1849

M a t e ri a l. D: $12.09 .2015-1$ sp., 08.04.2016 - 2 sp.

Depressaria a lbipuncte lla ([Denis et Schiffermüller], 1775)

M a t e r i a l . D: 26.06.2015, 13.05.2016, 27.06.2016, 04.05.2018 - 1 sp. per night. L: 20.04.2007, 20.06.2007, 16.03.2008, 18.04.2009, 20.03.2011, 16.04.2012, 01.03.2014, 02.03.2014, 07.03.2014, 22.03.2014, 20.10.2018, 28.10.2018, 17.03.2019, 15.09.2019, 01.03.2020, 07.03.2020, 10.07.2020 - 1-25 sp. per day, mostly under bark of trees.

\section{* E th mia vittalbella (Christoph, 1877) (Fig. 34)}

M a teria l. D: 09.05.2018-3 sp.

D i s t r i b u t i o n. Malta (Lepiforum, 2020), Russia: S and SE European part of Russia (Volga-Don, Low-Volga, Mid-Volga and South Ural regions), record from Primorskiy Krai needs verification (Dubatolov, Ustjuzhanin, Zintshenko, 1997; Sinev, Shovkoon, 2019), Morocco, Algeria, Tunisia, Lybia, Egypt, Palestine, Saudi Arabia, Turkmenistan, Uzbekistan, SE Kazakhstan, Iran, Iraq, Syria, Afghanistan, Armenia, NW China, Pakistan (Sattler, 1967).

\section{Eth mia quadrillella (Goeze, 1783)}

M a t e r i a l. D: 23.07.2008 - 1 sp. P: 24.05.2020 - 1 sp. on trunks of Quercus robur.

E th mia candide lla (Alphéraky, 1908)

M a t e r i a l. D: $21.09 .2012-2$ sp., 13.09.2013 - 1 sp., 29.08.2015 - 3 sp., 04.09.2015 - 8 sp., $12.09 .2015-1$ sp., 02.10.2015 - 1 sp., 31.08.2018 - 1 sp., 08.09.2018 - 1 sp., 14.09.2018 - 1 sp., 06.09.2019 - 1 sp., 14.09.2019 - 12 sp., $28.09 .2019-$ 1 sp., 21.08.2020 - 1 sp., 29.08.2020 - 1 sp., 11.09.2020 - 1 sp., 18.09.2020 - 5 sp. C: 01.09.2015, 24.09.2015, 28.09.2015, 30.09.2015 - 1 sp. per night. P: 11.09.2011 — 1 sp. ex. larva, 26.04.2014 — tens of larvae (1 imago hatched on 10.08.2014), 03.09.2016 3 sp., 11.09.2016 - 8 sp., 17.04.2016 - 1 larva, 18.04.2016 - 1 larva, 03.05.2016 - 1 larva, 03.09.2017 - 1 sp., 02.09 .2018 - 1 sp., 08.09.2019 - 20 sp. on trunks of Quercus robur. All larvae on Buglossoides czernjajevii.

\section{* Ethmia terminella Fletcher, 1938}

M a t e ri a l. D: $23.06 .2018-1$ sp. C: $23.06 .2018-1$ dead sp.

E th mia bipunctella (Fabricius, 1775)

M a t e ri a l . D: 15.09.2012, 28.04.2013, 14.08.2015, 29.08.2015, 04.09.2015, 03.06.2016, 23.06.2017, 04.08.2017, 09.05.2018, 17.08.2018, 11.05.2019, 01.05.2020, 24.07.2020, 21.08.2020 - 1-2 sp. per night. C: 30.05.2000, 23.07.2000, 04.06.2006, 27.08.2014, 30.08.2018 - 1 sp. per night.

\section{Family STATHM O P O I D A E Janse, 1917}

* Stathmopoda pedella (Linnaeus, [1760])

M a t e ri a l. P: 09.06.2018 - 1 sp., alder forest.

\section{Family C OLEOPHOR I D E Bruand, 1850}

* Plegmidia potentillae (Elisha, 1885) (Figs. 35-36)

M a t e ri a l. L: 27.10.2019 - 3 leaves with mines on Rubus caesius.

\section{F a mily M O M P H I D A Herrich-Schäffer, 1857}

* Mompha subbistrigella (Haworth, 1828)

M a t e r i a l. D: 10.05 .2014 - 1 sp., 17.05.2015 - 1 sp., 28.06.2015 - 1 sp., 04.09.2015 - 1 sp., 19.06.2016 - 3 sp., 23.06.2017 — 1 sp., 24.06.2018 — 1 sp. L: 02.03.2014 — 1 sp. under bark. 
* Mompha e pi lobi e lla ([Denis et Schiffermüller], 1775)

M a t e r i a l . D: 19.06 .2015 - 1 sp., 26.06.2015 - 10 sp., 03.07.2015 - 4 sp., 31.07.2015 - 1 sp., 27.06.2017 - 2 sp., 26.06.2020 - 1 sp., 03.07.2020 - 1 sp.

* Mompha ochraceella (Curtis, 1839)

M a t e r i a l. $\quad$ D: $12.06 .2015-1$ sp., 19.06.2015 - 2 sp., 19.06.2016 - 2 sp., $23.06 .2017-2$ sp., $27.06 .2017-3$ sp., 21.06.2019 - 1 sp., $12.06 .2020-1$ sp.

\section{F a m i l y C O S M O P T E R I G I D A E Heinemann et Wocke, 1876}

* Sorhagenia rhamniella (Zeller, 1839)

M a te ri a l. D: $12.06 .2015-1$ sp.

Pancalia leuwenhoekella (Linnaeus, [1760])

M a t e ri a l . L: 13.05.2012， 10.05.2014, 16.05.2014, 02.05.2016, 14.05.2017， 05.05.2019, 17.05.2019, 19.05.2019, 02.05.2020 - 1-2 sp. per day.

Pancalia nodosella (Bruand, 1850)

M a t e r i a l. P: $16.05 .2019-1$ o on flowers of Crataegus.

* Limnaecia phragmitella Stainton, 1851

M a t e ri a l . D: 26.06.2015 - 1 sp., 03.07.2015 - 3 sp., 10.07.2015 - 1 sp., 24.07.2015 - 1 sp., 19.06.2016 - 2 sp., 24.06.2016 - 1 sp., 27.06.2016 - 6 sp., 01.07.2016 - 1 sp., 27.06.2017 - 3 sp., 28.07.2017 - 1 sp., 03.07.2020 - 1 sp.

Vulcaniella grandiferella Sinev, 1986

M a t e r i a l. D: 03.06.2016 — 1 sp., $12.06 .2020-1$ sp.

Eteobalea anonymella (Riedl, 1965)

M a teria l. D: $19.06 .2016-1 \mathrm{sp}$

Pyroderces argyrogrammos (Zeller, 1847)

M a t e r i a l . D: 21.09.2012, 31.07.2015, 07.08.2015, 14.08.2015, 29.08.2015, 04.09.2015, 12.09.2015, 18.09.2015, 19.06.2016, 24.06.2016, 27.06.2016, 01.07.2016, 26.07.2016, 31.07.2016, 19.08.2016, 27.06.2017, 19.08.2017, 18.05.2018, 25.05.2018, 21.06.2019, 27.06.2019, 16.08.2019, 06.09.2019, 14.09.2019, 03.07.2020, 08.08.2020, 28.08.2020 — 1-3 sp. per night. L: 06.06.2018 — 1 sp.

\section{* Pyroderces klimeschi Rebel, 1938}

M a t e r i a l. D: $09.06 .2018-1$ sp., $24.06 .2018-1$ sp.

* Cosmopterix zieglerella (Hübner, [1810]) forest.

M a t e ri a l . P: 30.05.2020 - 1 sp., birch-alder forest, 30.08 .2020 and 13.09.2020 - 3 mines on Humulus lupulus, aspen-birch

* Cosmopterix scribaiella Zeller, 1850

M a t e ri a l. D: $27.06 .2016-1 \mathrm{sp}$

* Cosmopterix li enigi e lla Lienig et Zeller, 1846

M a t e ri a l . D: 23.05 .2015 - 1 sp., 30.05.2015 - 1 sp., 24.07.2015 - 2 sp., $13.05 .2016-1$ sp., 09.05.2018 - 1 sp., 18.05.2019-1 sp.

F a m i ly G E L E C H I I A E Stainton, 1854

* Aproaerema sangiella (Stainton, 1863)

M a t e r i a l. D: $27.05 .2016-1 \mathrm{sp}$

Aproaerema anthyllide lla (Hübner, [1813])

M a t e r i a l. D: 10.07.2015 — 1 sp., 02.10.2015 — 1 sp., 28.07.2017 — 1 đ.

Anacamps is populella (Clerck, 1759)

M a t e r i a l . D: 04.06.2012 - 1 sp., 27.06.2017 — 1 sp.

* Anacampsis blattariella (Hübner, 1796)

M a t e r i a l . P: 10.06.2018 - 1 sp., birch forest.

Anacamps is timidella (Wocke, 1887)

M a t e r i a l . D: 03.07.2015 - 1 sp., 31.07.2015 - 1 sp., 09.08.2019 — 1 sp. 
* Me s ophleps trinotella Herrich-Schäffer, 1856

M a t e ri a l. D: 03.08.2018 - 1 sp., 09.08.2019-1 sp.

* Sophronia sicariellus (Zeller, 1839)

M a teria l. P: 04.07.2017-1 sp.

Nothris verbascella ([Denis et Schiffermüller], 1775)

M a t e r i a l . D: 25.06.2016 — 1 sp., $13.06 .2020-1$ sp. C: $10.09 .2009-1$ sp. P: $07.06 .2020-1$ sp.

Anarsia lineatella Zeller, 1839 s. I.

M a t e r i a l . D: 12.05.2012, 19.06.2015, 04.09.2015, 27.05.2016, 03.06.2016, 19.06.2016, 23.06.2017, 27.06.2017, 25.05.2018, 14.09.2018, 07.08.2020, 26.06.2020, 21.08.2020 - 1-3 sp. per night.

* Anarsia eleagnella Kuznetsov, 1957

M a te ri a l. D: $19.08 .2017-1$ sp.

* Dichomeris dera sella ([Denis et Schiffermüller], 1775)

M a teria l. L: $24.05 .2017-1$ sp.

Dichomeris limosellus (Schläger, 1849)

M a t e r i a l . D: 23.06.2017 — 1 sp., 27.06.2017 — 1 sp., 14.09.2019 — 1 sp. C: 08.2014 — 1 sp. P: 01.06 .2017 — 1 sp. at dusk.

Dicho me ris rasile Ila (Herrich-Schäffer, [1854])

M a t e r i a l. D: 28.05.2012 - 1 sp., 28.06.2015 - 1 sp., 31.07.2015 - 1 sp., 19.06.2016 - 1 sp., $13.06 .2020-1$ sp.

Dichomeris al a cella (Zeller, 1839)

M a t e ri a l . D: 14.06.2012, 26.06.2015, 28.06.2015, 10.07.2015, 31.07.2015, 14.08.2015, 19.06.2016, 24.06.2016, 25.06.2016, 27.06.2016, 01.07.2016, 23.06.2017, 27.06.2017, 28.07.2017, 03.08.2018, 07.06.2019, 16.08.2019, 19.06.2020, 26.06.2020, 28.06.20201-6 sp. per night. C: 01.07.2012 - 1 sp. P: 25.06.2017, 06.07.2017, 23.07.2017, 08.07.2018, 08.06.2019, 07.07.2019, 13.07.2019, 28.07.2019 - 1-4 sp. per day on trunks of Quercus robur.

* A compsia cinerella (Clerck, 1759)

M a t e ri a l. P: $24.05 .2020-1 \mathrm{sp.}$

Brachmia dimidiella ([Denis et Schiffermüller], 1775)

M a t e ri a l . D: 28.05.2012, 10.07.2015, 01.07.2016, 19.08.2016, 23.08.2016, 27.06.2017, 31.08.2018, 13.06.2020, 03.07.2020 — 1 sp. per night. L: 06.06.2018 — 1 sp., 06.06.2019 — 1 sp.

* Brachmia inornatella (Douglas, 1850)

M a t e r i a l . D: 03.06.2016, 25.06.2016, 15.07.2016, 27.06.2017, 21.06.2019, 26.06.2020, 28.06.2020 - 1-3 sp. per night.

* Helcystogramma triannuleIla (Herrich-Schäffer, [1854])

M a t e r i a l. D: $15.09 .2012-1$ sp., 18.05.2018 - 1 sp., 03.07.2020 - 1 sp.

* Helcystogramma lutatella (Herrich-Schäffer, [1854])

M a t e r i a l . D: 31.07.2015 - 3 sp., 17.07.2016 - 3 sp., 20.07.2019 - 1 sp., 03.08.2019 — 1 sp., 29.08.2020 — 1 sp.

Helcystogramma arulensis (Rebel, 1929)

M a t e r i a l. D: 24.07 .2015 - 1 sp., 31.07.2015 - 1 sp., 25.06.2016 - 1 sp., 06.08.2016 - 1 sp., 27.06.2017 - 2 sp., 28.07.2017 - 2 sp., 04.08.2017 - 1 sp.

Pexicopia malvella (Hübner, [1805])

M a t e r i a l . D: 14.06.2012, 19.06.2015, 03.07.2015, 15.07.2015, 23.06.2017, 27.06.2017, 24.06.2018 — 1 sp. per night.

Chrysoesthia sexguttella (Thunberg, 1794)

M a t e r i a l . D: 31.08.2018 - 1 sp., 21.06.2019 — 1 sp., 07.06.2020 - 1 sp. in the daytime. C: 22.06 .2018 - 1 sp.

Bryotropha desertella (Douglas, 1850)

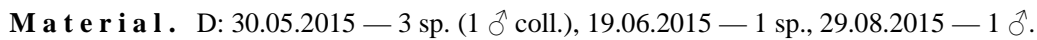

* Bryotropha affinis (Haworth, 1828)

M a t e ri a l. D: $26.07 .2016-1$ sp.

Aristotelia subericinella (Duponchel, [1843])

M a t e ri a l. D: 27.07.2018-2 sp. 
Megacraspedus Iongipa lpe lla Junnilainen, 2010

M a t e r i a l . D: 12.06.2015, 19.06.2015, 26.06.2015, 28.06.2015, 03.07.2015, 19.06.2016, 24.06.2016, 27.06.2016, 01.07.2016, 27.06.2017, 09.06.2018, 24.06.2018, 21.06.2019, 28.06.2020 - 1-12 sp. per night.

Ptocheusa inopella (Zeller, 1839)

M a t e ri a l. D: 04.05.2018 - 1 sp. P: 08.07.2018 - 1 sp.

Artemaea lonchoptera Staudinger, 1871

M a t e r i a l . D: 23.07.2008, 14.06.2012, 26.06.2015, 17.07.2016, 27.06.2017, 21.06.2019, 26.06.2020 - 1 sp. per night.

I s ophrictis striate lla ([Denis et Schiffermüller], 1775)

M a t e ri a l . D: 19.06.2015 - 1 sp., 10.07.2015 — 2 sp., 24.07.2015 - 1 sp.

* Apodia bifractella (Duponchel, [1843])

M a t e ri a l. D: 27.06.2016 - 1 sp., 01.07.2016 - 1 sp., 09.08.2019 - 1 sp.

Mo nochroa s epicole lla (Herrich-Schäffer, [1854])

M a t e ri a l. D: 24.07.2015 - 1 sp., 07.08.2015 - 1 sp.

* Monochroa palustrellus (Douglas, 1850)

M a t e ri a l. D: 01.07.2016 - 1 sp.

Monochroa hornigi (Staudinger, 1883)

M a t e ri a l. D: 26.06.2015 - 1 sp., 06.08.2016 - 2 sp.

* Oxypteryx wilkella (Linnaeus, 1758)

M a t e ri a l. D: 08.08.2020-1 sp.

* Athrips rancidella (Herrich-Schäffer, [1854])

M a t e r i a l. D: 19.06.2015 - 1 sp., 03.07.2015 - 1 sp., 01.07.2016 - 1 sp., $13.06 .2020-1$ sp.

Athrips nigricostella (Duponchel, [1843])

M a te ri a l. D: 10.05.2014 - 1 sp., 23.05.2015 - 1 sp., 27.05.2016-1 sp.

* Mirificarma lentiginosella (Zeller, 1839)

M a te ri a l. D: 04.09.2015 - 1 sp.

Aroga ve lo c e lla (Zeller, 1839)

M a t e ri a l . D: 28.06.2015, 03.07.2015, 23.06.2017, 27.06.2017, 28.07.2017, 19.08.2017, 04.05.2018, 09.06.2018, 17.08.2018, 07.06.2019, 12.06.2020 — 1-2 sp. per night. C: 06.2002 - 1 sp., 06.06.2014 - 1 sp. P: 08.05.2017, 27.04.2018, 27.04.2019, 04.05.2019, 18.04.2020 - 1-20 sp. per day.

* Ge lech i a rhombe lla ([Denis et Schiffermüller], 1775)

M a t e r i a l. P: $21.06 .2020-1$ sp. on trunks of Quercus robur.

Ge le chia s coti nella Herrich-Schäffer, [1854]

M a teria l . D: $08.08 .2020-1$ sp. P: 22.06.2014, 04.07.2017, 23.07.2017, 08.07.2018, 26.06.2019-1-10 sp. per day on trunks of Quercus robur.

* Ge lechia so rorcule lla (Hübner, [1817])

M a te ri a l. D: 17.07.2016-1 sp.

* Gelechia rhombelliformis Staudinger, 1871

M a t e r i a l. D: $14.08 .2015-1$ sp. P: $12.08 .2018-1$ sp. on trunks of Populus nigra.

* P s or ic optera gibbos ella (Zeller, 1839)

M a t e r i a l. P: $05.08 .2018-1$ sp. on trunks of Quercus robur.

* Scrobipalpa a cuminatella (Sircom, 1850)

M a t e ri a l. D: 07.08.2013 - 1 sp., 01.07.2016 - 1 sp., 13.08.2016 - 1 sp.

* S crobipalpa obsolete lla (Fischer von Röslerstamm, 1840)

M a t e ri a l. D: 10.07.2015 - 1 sp., 13.05.2016 - 3 sp., 27.05.2016 - 1 sp., 13.08.2016 - 1 sp.

* S c robipa Ipa indigne lla (Staudinger, 1879)

M a te ri a l. D: $23.05 .2015-1$ sp. 
S. O. DEMYANENKO, O. V. BIDZILYA, E. A. KAROLINSKIY New records of Lepidoptera (Insecta) of Severodonetsk (Luhansk Region, Ukraine) and its environs

S c robipa lpa atrip lice lla (Fischer von Röslerstamm, 1840)

M a te ri a l. D: $30.04 .2018-1$ sp.

S crobipalpa ocellatella (Boyd, 1858)

M a t e ri a l. D: 21.09.2012 - 1 sp., 13.09.2013 - 1 sp., 12.09.2015 - 3 sp., 18.09.2015 - 3 sp., 25.09.2015 - 2 sp., 14.09.2019-1 sp.

S c robipalpa salinella (Zeller, 1847)

M a t e ri a l. D: $24.05 .2014-1$ sp., 31.08.2018 - 1 sp.

* Caryocolum fischerella (Treitschke, 1833)

M a te ri a l. D: 09.08.2019-1 sp.

* Caryocolum proxima (Haworth, 1828)

M a t e r i a l. D: 07.08.2013 - 1 sp., 24.07.2015 — 2 sp., 07.08.2015 - 3 sp., 03.08.2019 — 1 sp., 08.08.2020 — 1 sp.

* Te le i odes l u cule lla (Hübner, [1813])

M a t e ri a l . P: 23.07.2017 - 1 sp., 06.05.2018 - 1 sp., $09.05 .2018-6$ sp., 28.05.2018 - 4 sp., $11.05 .2019-4$ sp., 16.05.2019 — 1 sp., 31.05.2019 — 3 sp., 01.06.2019 — 1 sp., 26.06.2019 — 1 sp., on trunks of Quercus robur.

* Carpatolechia a enigma (Sattler, 1983)

M a t e ri a l . P: 08.05.2017 - 1 sp., 23.07.2017 - 3 sp., 05.08.2018 - 1 sp., $11.05 .2019-4$ sp., 16.05.2019 - 1 sp., 31.05.2019 - 3 sp., 08.06.2019 - 3 sp., 13.07.2019 - 1 sp., on trunks of Quercus robur.

* Carpatolechia fugacella (Zeller, 1839)

M a t e r i a l . D: 18.09.2015, 03.07.2016, 23.06.2017, 27.06.2017, 19.08.2017, 24.06.2018, 20.07.2018, 13.06.2020, 19.06.2020, 28.06.2020 - 1-3 sp. per night. C: 13.06 .2018 - 1 sp., 10.07.2018 - 1 sp. P: 09.08.2016, 23.07.2017, 08.06.2019, 26.06.2019, 05.07.2020 - 1-5 sp. per day on trunks of Quercus robur and Populus nigra.

* Ps eudotelphusa scalella (Scopoli, 1763)

M a t e r i a l. P: 25.06.2017 — 1 sp., 21.06.2020 — 1 sp., on trunks of Quercus robur.

* Streyella anguinella (Herrich-Schäffer, 1861)

M a te ria l. D: $24.05 .2014-1$ sp.

* Altenia scriptella (Hübner, 1796)

M a t e r i a l. D: 26.05.2017 — 1 sp. L: 22.06.2019 — 3 sp., 08.06.2020 — 1 sp., 03.07.2020 — 2 sp., on trunks of trees.

Re curvaria nane Ila ([Denis et Schiffermüller], 1775)

M a t e r i a l . D: 07.08.2013, 19.06.2015, 26.06.2015, 03.07.2015, 10.07.2015, 17.07.2015, 24.07.2015, 07.08.2015, 14.08.2015, 19.06.2016, 25.06.2016, 08.07.2016, 26.07.2016, 06.08.2016, 23.06.2017, 27.06.2017, 28.07.2017, 04.08.2017, 19.08.2017, 24.06.2018, 06.07.2018, 31.08.2018, 07.09.2019, 21.06.2019, 03.08.2019, 09.08.2019, 16.08.2019, 26.06.2020, 08.08.2020, 14.08.2020 - 1-6 sp. per night; 28.10.2018 - 3 mines on Malus domestica and 3 mines on Prunus domestica, 03.11.2018 - 2 mines on Malus domestica, 28.09.2019 - 6 mines on Prunus cerasus, 19.09.2020 - 2 mines on Prunus cerasus and 1 mine on Prunus domestica, 31.10.2020 - 1 mine on Prunus armeniaca, all empty. C: 06.08.2015 - 1 sp. L: 23.07.2017 — 1 sp., 08.06.07.2018 — 1 sp., 08.07 .2018 - 2 sp., 19.07 .2018 1 sp., on trunks of Pyrus and Quercus robur; 26.08.2018 and 06.10.2019 — empty mines on Malus.

* Recurvaria leucatella (Clerck, 1759)

M a t e r i a l . P: 27.05 .2018 - 1 sp., 08.06.2018 - 1 sp., 09.06.2018 - 3 sp., 31.05.2019 - 1 sp., 07.07.2019 - 1 sp., on trunks of Pyrus.

* Ste nolechia gem mella (Linnaeus, 1758)

M a t e ri a l. P: 02.09.2018 - 1 sp. on trunks of Quercus robur.

S uperfamily AL UCITO IDEA Leach, 1815

Family ALUCITIDAE Leach, 1815

* Alucita grammodactyla Zeller, 1841

M a t e ri a l. D: $31.07 .2015-1$ sp., 29.08.2020 - 1 sp. C: $30.07 .2008-1$ sp.

S uperfamily PTEROPHOROIDEA Latreille, 1802

Family PTEROPHORIDAE Latreille, 1802

Agdistis adactyla (Hübner, [1819])

M a t e ri a l. D: $03.07 .2015-1$ sp., 31.08.2018 — 1 sp. P: $21.07 .2018-1$ ㅇ. 
* Amblyptilia acanthadactyla (Hübner, [1813])

M a t e ri a l. C: $29.08 .2009-1 \mathrm{sp.}$

Cna emidophorus rhododactyla ([Denis et Schiffermüller], 1775)

M a t e r i a l . D: 19.06.2016, 25.06.2016, 23.06.2017, 27.06.2017, 09.06.2018, 21.06.2019, 28.06.2020 - 1 sp. per night.

* Oxyptilus distans (Zeller, 1847)

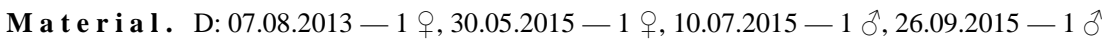

* Ge ina didactyla (Linnaeus, 1758)

M a t e r i a l . L: 15.06.2000, 30.05.2006, 06.06.2006, 31.05.2007, 04-17.06.2007, 06.06.2019-1-25 sp. per day.

Pterophorus pentadactyla (Linnaeus, 1758)

M a t e ri a l. $\quad$ D: 31.07 .2015 - 1 sp., 06.08 .2016 - 1 sp., 09.06.2018 - 1 sp. L: 11.06.2001, 31.05.2007, 04.06.2007, 06.06.2007, 31.05.2008 - 1-2 sp. per day.

* Merrifieldia tridactyla (Linnaeus, 1758)

M a t e r i a l . P: 19.06.2016, 27.06.2016, 22.06.2017, 25.06.2017, 08.06.2019 - 1-10 sp. per day on Thymus pallasianus.

* Hellinsia inulae (Zeller, 1852)

M a t e r i a l . D: 30.05.2015 — 1 sp., 06.08.2017 — 1 sp.

Hellinsia osteodactylus (Zeller, 1841)

M a t e r i a l . D: 01.09.2007, 10.07.2015, 07.08.2015, 14.08.2015, 27.05.2016, 19.06.2016, 13.08.2016, 19.08.2017, 06.07.2018, 27.07.2018, 17.08.2018, 07.06.2019, 09.08.2019, 21.08.2020 — 1-7 sp. per night.

Emmelina monodactyla (Linnaeus, 1758)

M a t e r i a l. D: $15.08 .2006,20.08 .2006,23.07 .2008,07.08 .2013,11.10 .2014,28.06 .2015,03.07 .2015,14.08 .2015,04.09 .2015$, 02.10.2015, 03.06.2016, 24.06.2016, 17.07.2016, 06.08.2016, 28.07.2017, 04.08.2017, 19.08.2017, 06.07.2018, 17.08.2018, 09.08.2019, 03.07.2020 - 1-5 sp. per night.

S uperfamily EPERMENIOIDEA Spuler, 1910

Family EPERME N I I A E Spuler, 1910

* Epermenia illigerella (Hübner, [1813])

M a t e ri a l. D: $19.06 .2016-1 \mathrm{sp.}$

Ochromolopis zagulajevi Budashkin et Satschkov, 1991

M a t e r i a l . $\quad$ D: $\quad 31.07 .2015, \quad 19.06 .2016,25.06 .2016,27.06 .2016, \quad 17.07 .2016, \quad 27.06 .2017, \quad 21.06 .2019, \quad 28.06 .2020$, 31.07.2020 - 1-2 sp. per night.

S uperfamily CHOREUTO IDEA Stainton, 1858

Family CHOREUTIDAE Stainton, 1858

* Millieria dolosalis (Heydenreich, 1851)

M a t e r i a l. L: 18.05.2011 — 1 sp., 18.05.2014 — 2 sp., 10.05.2016 — 2 sp., 17.05.2019 — 1 sp., 19.05.2019 — 2 sp.

Superfamily C O S O IDEA Leach, 1815

Family C O S I D A E Leach, 1815

Cossus cossus (Linnaeus, 1758)

M a t e r i a l . $\quad$ D: 19.05 .2012 - 1 sp., 28.05.2012 - 1 sp., 03.06.2016 - 2 sp., 23.06.2017 - 1 sp., 19.06.2020 - 1 sp. L: 31.03.2007 — 1 dead larva, 10.03.2008 — 1 larva, 16.03.2008 — 1 larva, 12.04.2009 — 1 larva, under bark of Quercus robur.

Parahypopta c a estrum (Hübner, [1808])

M a t e r i a l. D: $24.05 .2014-1$ sp., 30.05.2015 - 1 sp.

Dyspessa salicicola (Eversmann, 1848)

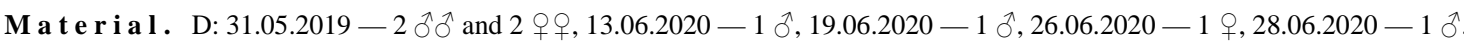

Zeuzera pyrina (Linnaeus, [1760])

M a t e r i a l . D: $27.07 .2000-1$ sp., 30.07.2000 - 1 sp., 26.06.2015 - 1 sp., $31.07 .2020-1$ sp. 
Phragmataecia castaneae (Hübner, [1790])

M a t e r i a l. D: $07.07 .2007,12.07 .2007,15.07 .2007,20.07 .2007,28.07 .2007,23.07 .2008,11-13.05 .2012,19.05 .2012$, 28.05.2012, 22.06.2012, 11.05.2013, 16.05.2014, 24.05.2014, 12.06.2015, 26.06.2015, 28.06.2015, 10.07.2015, 03.06.2016, 05.06.2016, 19.06.2016, 24.06.2016, 27.06.2016, 01.07.2016, 03.07.2016, 17.07.2016, 31.07.2016, 26.05.2017, 23.06.2017, 27.06.2017, 25.05.2018, 09.06.2018, 24.06.2018, 06.07.2018, 20.07.2018, 27.07.2018, 18.05.2019, 24.05.2019, 31.05.2019, 07.06.2019, 21.06.2019, 05.07.2019, 07.06.2020, 12.06.2020, 19.06.2020, 26.06.2020, 03.07.2020, 18.07.2020 - 1-10 sp. per night.

\section{F a mi l y S E S I I D A E Boisduval, 1828}

S e sia ap iformis (Clerck, 1759)

M a t e r i a l. C: 1996-1997 — common, 09.06.2015 — 1 sp., in the daytime near Populus.

Paranthrene tabaniformis (Rottemburg, 1775)

M a t e r i a l. C: 1996-1997 — 1 sp., in the daytime near Populus.

Synanthedon myopaeformis (Borkhausen, 1789)

M a teria l. D: $15.06 .2012-1 \mathrm{sp.}$

Synanthedon tipuliformis (Clerck, 1759)

M a t e r i a l . D: 03.07.2017 - 1 ô, in the daytime, $12.06 .2020-1$ sp., in the daytime.

* Pyropteron triannuliformis (Freyer, 1843)

M a t e r i a l. D: 30.05.2015 - 1 sp., in the daytime, fresh imago on Rumex acetosa.

Superfamily Z Y G A E N O D E A Latreille, 1809

F a mily L IMA C O D I A E Duponchel, 1845

Apoda limacodes (Hufnagel, 1766)

M a t e r i a l . D: $12.07 .2007-1$ sp., 20.07.2007 — 1 sp., 11.08.2007 — 1 sp., 10.07.2015 — 1 sp., 28.07.2017 — 1 sp.

F amily Z Y G A E N I A E Latreille, 1809

* Jordanita chloros (Hübner, [1813])

M a t e ri a l. L: 03.07.2020 - 1 sp., 18.07.2020 - 1 sp.

Jordanita graeca (Jordan, 1907)

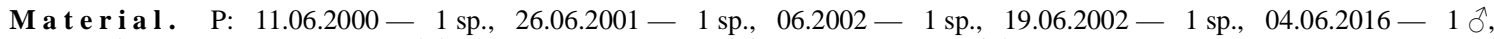

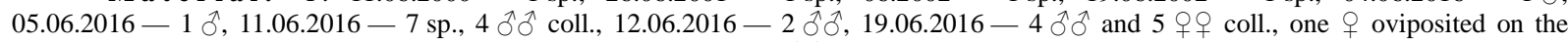
underside of a leaf of Helichrysum arenarium, 24.06.2016 - 2 के 0 , 22.06.2017 - 1 sp., 25.06.2017 — 2 sp., 04.07.2017 - 1 sp., 06.07.2017 - 1 ơ, 09.06.2018 - 1 sp., 10.06.2018 - 1 sp., 31.05.2019 - 1 sp., 05.07.2020 - 1 ㅇ.

\section{Zygaena centaureae Fischer von Waldheim, 1832}

M a t e r i a l . L: 04-14.06.2007 (1 imago hatched on 09.07.2007), 11.06.2011 (4 imago hatched on 02-06.07.2011), 01.06.2017, 03.06.2018, 06.06.2018, 22.06.2019 - 1-10 larvae per day on Apiaceae (probably Silaum silaus); 10.07.2020 — 3 sp., 18.07.2020 — 1 sp., 26.07.2020 - $1 \mathrm{sp.}$

Zygaena filipendulae (Linnaeus, 1758)

M a t e r i a l . L: 06.1998, 04.07.2006, 15.07.2006, 30.06.2007, 05.07.2007, 14-18.07.2011, 14.06.2012, 03.07.2016, 06.06.2018, 10.07.2020 - 1-100 sp. per day.

Superfamily PAP I L I O N O I D E A Latreille, 1802

Family PAP ILION I A E Latreille, 1802

Parnassius mnemosyne (Linnaeus, 1758)

M a t e ri a l. P: $11.05 .2020-5 \delta^{\Uparrow}\left(48.9637^{\circ} \mathrm{N}, 38.6045^{\circ} \mathrm{E}\right)$.

Family H E S P ER I I D A Latreille, 1809

Pyrgus armoricanus (Oberthür, 1910)

M a t e r i a l. P: 18.08.2013 - 2 sp., 13.09.2016 — 2 sp., 13.09.2017 - 1 sp., 16.09.2018 - 1 sp.

Family P I ER I D A E Swainson, 1820

Colias myrmidone (Esper, [1781])

M a teri a l. P: 1996-1997-1 §. 


\section{Family LY C A E N I A E Leach, 1815}

Satyrium acaciae (Fabricus, 1787)

M a t e r i a l . P: $21.06 .2020-1$ sp. $\left(48.9646^{\circ} \mathrm{N}, 38.6005^{\circ} \mathrm{E}\right)$.

Plebejus idas (Linnaeus, 1761)

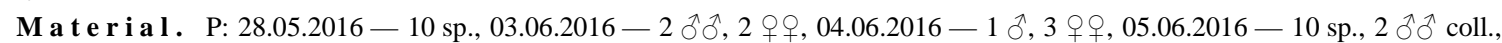
11.06.2016 - 3 sp., 28.05.2018 - 1 sp., 01.06.2019 - 1 sp., 28.08.2020 - 1 ふे, $13.09 .2020-1$ ㅇ.

F a mily N Y M P H A L I D A E Rafinesque, 1815

A rethusana arethusa ([Denis et Schiffermüller], 1775)

M a t e r i a l . P: $19.07 .2020-1$ sp. $\left(49.0187^{\circ} \mathrm{N}, 38.5155^{\circ} \mathrm{E}\right), 02.08 .2020-1 \mathrm{sp}$., at the same place.

Hyp n ephe le lupina (Costa, 1836)

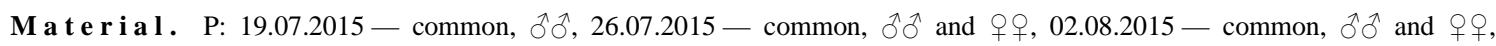
09.08.2015 - 1 sp., 11.06.2016 - 2 sp., 27.06.2016 - 1 sp., 22.06.2017 — 3 sp., 04.07.2017, 06.07.2017, 10.06.2018, 14.07.2018,

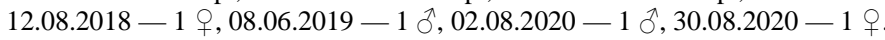

F a b r iciana niobe (Linnaeus, 1758)

M a t e ri a l . P: $08.06 .2018-1$ sp. $\left(48.9216^{\circ} \mathrm{N}, 38.5398^{\circ} \mathrm{E}\right)$.

Boloria euphrosyne (Linnaeus, 1758)

M a t e r i a l . P: $07.06 .2020-1$ sp. $\left(48.9692^{\circ} \mathrm{N}, 38.5658^{\circ} \mathrm{E}\right)$.

Euphydryas aurinia (Rottemburg, 1775)

M a t e r i a l . L: $17.05 .2019-1$ sp. $\left(48.9132^{\circ} \mathrm{N}, 38.4869^{\circ} \mathrm{E}\right)$.

Me li ta e a trivia ([Denis et Schiffermüller], 1775)

M a te ri a l. P: $1997-1$ sp.

S uperfamily PYRAL O I D A Latreille, 1809

Family PYRALIDAE Latreille, 1809

* Delplanqueia ins criptella (Duponchel, 1836)

M a t e ri a l. D: 09.06.2018-1 $\delta$.

Phycita meliella (Mann, 1864)

M a t e ri a l. D: $24.07 .2015-1$ ઈ.

Ancylos is sareptalla (Herrich-Schäffer, 1861)

M a t e ri a l. P: $15.05 .2007-1$ sp.

* Assara terebrella (Zincken, 1818)

M a t e ri a l . D: $12.06 .2020-1$ sp.

* Phycitodes inquinatella (Ragonot, 1887)

M a t e ri a l. D: $18.07 .2020-1 \hat{\sigma}$.

* Cadra figuli le l la (Gregson, 1871)

M a t e r i a l . D: $17.08 .2018-1 \hat{\jmath}, 14.09 .2019-1 \hat{\jmath}, 26.06 .2020-1 \mathrm{sp}$.

F a mi l y C R A M B I D A E Latreille, 1810

* S coparia subfusca Haworth, [1811]

M a t e r i a l . D: 28.05.2012, 30.05.2015, 29.08.2015, 12.09.2015, 18.09.2015, 25.09.2015, 26.09.2015, 27.05.2016, 03.06.2016, 05.06.2016, 19.08.2016, 23.08.2016, 27.08.2016, 26.05.2017, 27.06.2017, 18.05.2018, 02.06.2018, 14.09.2018, 31.05.2019, 21.06.2019, 09.08.2019, 07.06.2020, 18.09.2020 - 1-5 sp. per night.

\section{* S coparia basistrigalis Knaggs, 1866}

M a t e r i a l . P: 21.06.2020 - 1 sp. on trunks of Quercus robur.

S c oparia pyra le lla ([Denis et Schiffermüller], 1775)

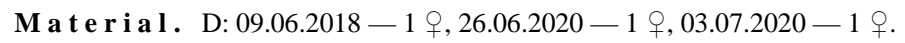




\section{* Eudonia lacustrata (Panzer, 1804)}

M a t e r i a l . D: $23.07 .2008-1$ sp., 12.06.2015 - 1 sp., 19.06.2015 - 1 sp., 26.06.2015 - 1 sp., 28.06.2015 - 2 sp., 10.07.2015 - 1 sp., 19.06.2016 - 2 sp., 24.06.2016 - 2 sp., 27.06.2016 - 1 sp., 26.07.2016 - 1 sp., 23.06.2017 — 3 sp., $27.06 .2017-$ 5 sp., 28.07.2017 - 2 sp., 04.08.2017 - 1 sp., 24.06.2018 - 1 sp., 06.07.2018 - 1 sp., 19.06 .2020 - 1 sp., $26.06 .2020-1$ sp. C: 22.05.2009 — 1 sp. L and P: 25.06.2017, 23.07.2017, 08.06.2019, 07.06.2020, 21.06.2020, 26.06.2020, 03.07.2020 — 1-15 sp. per day, on trunks of Quercus robur.

\section{* Eudonia mercurella (Linnaeus, 1758)}

M a t e r i a l. D: $\quad$ D: 24.07.2015 - 1 sp., 28.07.2017 - 1 sp., 27.07.2018 - 1 o, 21.06.2019- 1 sp., 05.07.2019- 1 sp., 20.07.2019 - 2 sp., 09.08.2019 - 1 sp., 03.07.2020 - 1 sp., 18.07.2020 - 1 +, 08.08.2020 - 1 sp. L and P: 08.07.2018 - 1 sp., 28.07.2018 - 4 sp., 12.08.2018 - 1 sp., 07.07.2019 - 1 sp., on trunks of Quercus robur.

\section{Chilo phragmitella (Hübner, [1810])} night.

M a t e r i a l . D: 15.07.2007, 20.07.2007, 14.08.2013, 28.07.2017, 27.07.2018, 03.08.2019, 28.06.2020, 24.07.2020 — 1 sp. per

\section{Chilo christophi Błezyński, 1965}

M a teri a l . D: $13.05 .2016-1$ ㅇ, 04.05.2018 - 1 o, 2 ㅇ, 09.05.2018- 1 o, $18.05 .2019-1$ ㅇ, $01.05 .2020-3$ sp. C: 08.05.2019-1 sp.

\section{Friedlanderia cicatric ella (Hübner, [1824]) \\ Material. D: $20.07 .2007-1$ sp.}

\section{Calamotropha paludella (Hübner, [1824])}

M a t e r i a l . D: 07.07.2007, 12.07.2007, 27.07.2007, 28.07.2007, 11.08.2007, 16.08.2007, 23.07.2008, 06.08.2008, 20.08.2008, 12.05.2012, 14.06.2012, 22.06.2012, 27.07.2012, 07.08.2013, 14.08.2013, 26.06.2015, 03.07.2015, 10.07.2015, 24.07.2015, 31.07.2015, 05.06.2016, 19.06.2016, 24.06.2016, 25.06.2016, 27.06.2016, 01.07.2016, 03.07.2016, 15.07.2016, 17.07.2016, 26.07.2016, 31.07.2016, 06.08.2016, 13.08.2016, 27.08.2016, 27.06.2017, 28.07.2017, 09.06.2018, 06.07.2018, 27.07.2018, 17.08.2018, 31.05.2019, 07.06.2019, 21.06.2019, 20.07.2019, 09.08.2019, 16.08.2019, 19.06.2020, 28.06.2020, 24.07.2020, 08.08.2020, 14.08.2020, 15.08 .2020 - 1-8 sp. per night. L: 04.08.2006 — 1 sp., 15.08 .2006 — 1 sp.

\section{* Calamotropha a ureliellus (Fischer von Röslerstamm, 1841)}

M a t e r i a l . D: 14.06.2012, 26.06.2015, 28.06.2015, 03.07.2015, 10.07.2015, 24.07.2015, 19.06.2016, 24.06.2016, 25.06.2016, 27.06.2016, 01.07.2016, 15.07.2016, 23.06.2017, 27.06.2017, 28.07.2017, 09.06.2018, 07.06.2019, 21.06.2019, 27.06.2019, 26.06.2020, 28.06.2020, 03.07.2020, 31.07.2020 - 1-6 sp. per night.

\section{Euchromius ocellea (Haworth, [1811])}

M a t e r i a l . D: 06.09.2008, 15.09.2012, 21.09.2012, 06.10.2012, 14.08.2013, 13.09.2013, 26.06.2015, 28.06.2015, 14.08.2015, 04.09.2015, 18.09.2015, 25.09.2015, 17.07.2016, 19.08.2016, 23.08.2016, 09.09.2016, 27.06.2017, 28.07.2017, 19.08.2017, 09.09.2017, 27.07.2018, 17.08.2018, 31.08.2018, 08.09.2018, 14.09.2018, 21.09.2018, 07.06.2019, 03.08.2019, 09.08.2019, 06.09.2019, 14.09.2019, 28.09.2019, 04.10.2019, 13.10.2019, 12.06.2020, 19.06.2020, 26.06.2020, 28.06.2020, 08.08.2020, 14.08.2020, 21.08.2020, 28.08.2020, 29.08.2020, 11.09.2020, 18.09.2020, 16.10.2020 - 1-8 sp. per night. C: $17.09 .2011-1$ sp., 08.06.2019 — 1 sp. P: $26.08 .2018-1$ sp., 02.09.2018-2 sp.

\section{* Euchromius gratiosella (Caradja, 1910)}

M a terial. D: $12.06 .2020-2 \hat{\jmath} \hat{\delta}$.

Euchromius jaxartella (Erschoff, 1874)

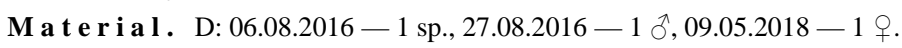

\section{Chrysoteuchia culmella (Linnaeus, 1758)}

M a t e r i a l . D: 12.06.2015, 26.06.2015, 28.06.2015, 03.07.2015, 10.07.2015, 17.07.2015, 19.06.2016, 24.06.2016, 27.06.2016, 01.07.2016, 23.06.2017, 27.06.2017, 06.07.2018, 31.05.2019, 21.06.2019, 05.07.2019, 20.07.2019, 12.06.2020, 13.06.2020, 19.06.2020, 26.06.2020, 28.06.2020, 03.07.2020 - 1-10 sp. per night. L and P: 15.07.2006, 31.05.2007, 13.06.2007, 20.06.2007, 14.06.2012, 05.06.2016, 07.06.2016, 08.05.2017, 14.05.2017, 22.06.2017, 03.06.2018, 06.06.2018 - 1-10 sp. per day.

\section{Crambus pascuella (Linnaeus, 1758)}

M a t e ri a l. D: 25.06.2016 - 1 sp., 27.06.2016 - 1 sp., 23.06.2017 - 2 sp., 06.07.2018 - 1 sp., 07.06.2019 - 1 sp., 27.06.2019 — 1 sp. L: 12.06.2006, 31.05.2007, 11.06.2011, 14.06.2012, 07.06.2016, 06.06.2018 - 1-2 sp. per day.

\section{Crambus pratella (Linnaeus, 1758)}

M a t e r i a l . D: 24.05.2014, 12.06.2015, 05.06.2016, 19.06.2016, 24.06.2016, 25.06.2016, 27.06.2016, 23.06.2017, 27.06.2017, 25.05.2018, 09.06.2018, 07.06.2019, 07.06.2020 - 1-8 sp. per night. P: 01.06.2006, 01.07.2006, 15.07.2006, 13.08.2006, 20.05.2007, 22.05.2010, 29.05.2010, 15.09.2013, 05.06.2015, 11.06.2015, 04.06.2016, 28.05.2017, 22.06.2017, 25.06.2017, 04.07.2017, 12.09.2017, 08.06.2018, 07.06.2020, 21.06.2020, 26.06.2020 - 1-50 sp. per day.

\section{Crambus lathoniellus (Zincken, 1817)}

M a t e r i a l . L: 30.05.2006, 26.05.2007, 29.05.2007, 04.06.2007, 22.05.2008 — 1-3 sp. per day. 


\section{* Crambus hamella (Thunberg, 1794)}

M a t e r i a l. D: $14.08 .2015-1$ sp., $04.09 .2015-2$ sp., $23.08 .2016-12$ sp., $27.08 .2016-1$ sp., $31.08 .2018-5$ sp. P: 28.08.2016, 02.09.2018, 08.09.2019 - 5-20 sp. per day.

Crambus perlella (Scopoli, 1763)

M a t e r i a l. D: $23.07 .2008-1$ sp., 10.07.2015 — 1 sp. P: 14.06.2007 — 1 sp.

\section{Agriphila de li ella (Hübner, [1813])}

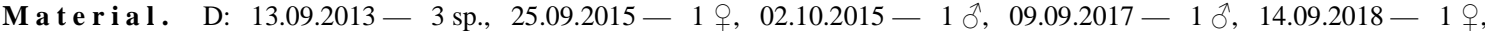
18.09.2020 - 1 우. P: $30.08 .2020-1$ 우.

\section{* Agriphila a eneociliella (Eversmann, 1844)}

M a t e ri a l. P: $16.09 .2018-1$ ô, $30.08 .2020-5$ sp.

\section{Agriphila straminella ([Denis et Schiffermüller], 1775)}

M a t e ri a l . $\quad$ D: 31.07 .2015 - 1 sp., 26.07.2016 - 1 sp., 31.07.2016 - 2 sp., 06.08.2016 - 1 sp., $04.08 .2017-1$ sp. L: 25.07.2016 — 15 sp., 18.07.2020 - 25 sp., 09.08.2020 - 25 sp.

\section{Agriphila poliellus (Treitschke, 1832)}

M a t e r i a l . $\quad$ D: 15.09 .2012 - 1 sp., 13.09.2013 - 3 sp., 12.09.2015 - 1 sp., 18.09.2015 - 2 sp., 08.09.2018 - 1 sp., 14.09.2018 - 1 sp., 14.09.2019 - 3 sp., 11.09.2020 - 1 sp. P: 11.09.2016 - 1 sp., 16.09.2018 - 1 sp.

\section{Catoptria pinella (Linnaeus, 1758)}

M a t e r i a l . D: 26.06.2015, 10.07.2015, 03.07.2016, 08.07.2016, 17.07.2016, 26.07.2016, 23.06.2017, 27.06.2017, 28.07.2017, 24.06.2018, 27.06.2019, 12.06.2020, 19.06.2020 - 1-3 sp. per night. P: 05.06.2000, 11.06.2000, 23.07.2000, 05.07.2001, 26.06.2020 1-20 sp. per day.

\section{Cat optria fulgide lla (Hübner, [1813])}

M a t e r i a l . $\quad$ D: 27.08 .2016 - 1 sp., 08.09.2018 - 1 sp., 15.08 .2020 - 1 sp., 21.08.2020 - 4 sp., $28.08 .2020-1$ sp., 29.08.2020 — 1 sp. P: 08.09.2018 — 1 sp., 08.09.2019 - 1 sp., 30.08.2020 - 2 sp. C: $26.08 .2016-1$ sp.

\section{Ca toptria fals e lla ([Denis et Schiffermüller], 1775)}

M a t e r i a l . D: 07.07.2007, 16.08.2007, 23.07.2008, 29.07.2008, 22.06.2012, 14.08.2013, 10.07.2015, 17.07.2015, 31.07.2015, 07.08.2015, 04.09.2015, 24.06.2016, 01.07.2016, 03.07.2016, 06.08.2016, 23.08.2016, 23.06.2017, 27.06.2017, 21.07.2017, 04.08.2017, 19.08.2017, 24.06.2018, 06.07.2018, 17.08.2018, 31.08.2018, 21.06.2019, 27.06.2019, 05.07.2019, 20.07.2019, 16.08.2019, 26.06.2020, 24.07.2020, 08.08.2020, 14.08.2020, 15.08.2020, 21.08.2020, 28.08.2020, 29.08.2020 - 1-3 sp. per night. C: $16.08 .2014-1$ sp., 21.06.2015 - 2 sp., 08.07.2016 — 1 sp., 06.08.2016 — 1 sp., 13.08.2016 - 1 sp., 31.07.2017 — 1 sp., 17.08.2017 — 1 sp.

\section{Catoptria verellus (Zincken, 1817)}

M a t e ri a l. D: $28.06 .2020-1$ sp.

Catoptria lythargyrella (Hübner, 1796)

M a t e r i a l. D: 13.09 .2013 - 1 sp., 04.09 .2015 - 1 sp., 12.09.2015 - 1 sp., 09.09 .2017 - 1 sp. P: 11.09 .2016 - 1 sp., 16.09.2018-1 sp.

\section{Xanthocrambus saxonellus (Zincken, 1821)}

M a t e r i a l. D: 28.06.2015 - 1 sp., 23.06.2017 - 1 sp., 28.06.2020 - 1 sp.

\section{Chrysocrambus linetella (Fabricius, 1781)}

M a t e r i a l . D: 24.05.2014, 04.06.2015, 12.06.2015, 19.06.2015, 05.06.2016, 19.06.2016, 24.06.2016, 25.06.2016, 27.06.2016, 01.07.2016, 23.06.2017, 27.06.2017, 18.05.2018, 25.05.2018, 09.06.2018, 24.06.2018, 06.07.2018, 24.05.2019, 31.05.2019, 07.06.2019, 21.06.2019, 27.06.2019, 12.06.2020, 13.06.2020, 19.06.2020 - 1-15 sp. per night. L and P: 27.05.2000, 01.06.2000, 03.06.2000, 06.06.2000, 10.06.2000, 11.06.2001, 12.06.2001, 17.06.2001, 01.07.2001, 29.04.2002, 10.05.2002, 12.05.2002, 16.05.2002, 17.05.2002, 27.05.2002, 21.06.2002, 04.05.2003, 18.05.2003, 10.05.2004, 16.05.2004, 12.05.2005, 16.05.2005, 04.06.2007, 06.06.2007, 11.06.2008, 28.06.2008, 02.07.2011, 03.06.2016, 07.06.2016, 27.05.2017, 06.06.2018, 06.06.2019 - 1-10 sp. per day.

\section{Chrysocramboides craterella (Scopoli, 1763)}

M a t e ri a l . D: 30.05.2015 - 1 sp., 12.06.2015 - 1 sp., 01.07.2016 - 1 sp., 18.05.2018 - 1 sp., 09.06.2018 - 1 sp., 24.05.2019 — 1 sp., 31.05.2019 - 2 sp., 07.06.2019 - 1 sp.

\section{Thisanotia chrysonuchella (Scopoli, 1763)}

M a t e ri a l . D: 10.05 .2014 - 1 sp., 16.05.2014 - 1 sp., 26.05.2017 - 1 sp., 09.05 .2018 - 2 sp., $18.05 .2019-1$ sp., 01.05.2020 - 1 sp. C: 12.05 .2005 - 1 sp. L: 20.05.2007 - 1 sp., 17-18.05.2014 - 1 sp., 02.05.2016 - 1 sp. P: 04.05.2000, 09.05.2000, 20.05.2000, 23.05.2000, 19.05.2001, 18.05.2006, 16.05.2007, 22.05.2007, 10.05.2010, 22.05.2010, 29.05.2010, 22.05.2015, 14.05.2017, 28.05.2017, 25.06.2017, 05.05.2018, 27.05.2018, 04.05.2019, 11.05.2019, 17.05.2019, 26.04.2020, 03.05.2020, 11.05.2020, 24.05.2020, 30.05.2020, 31.05.2020, 07.06.2020 - 1-20 sp. per day. 


\section{Pediasia jucunde ll us (Herrich-Schäffer, [1848])}

M a t e ri a l. P: $02.09 .2018-1 \hat{\jmath}, 4$ 우은

\section{P edia s ia l u te e lla ([Denis et Schiffermüller], 1775)}

M a t e r i a l . D: 28.05.2012, 12.06.2015, 26.06.2015, 10.07.2015, 19.06.2016, 25.06.2016, 27.06.2016, 27.06.2017, 21.07.2017, 24.06.2018, 06.07.2018, 24.05.2019, 28.06.2020 - 1-6 sp. per night. C: $30.05 .2012,07.06 .2012,11.06 .2015,17.06 .2015,18.06 .2015$, 21.06.2015 - 1-5 sp. per night. L and P: 22.05.2005, 25.05.2005, 06.06.2006, 13.08.2006, 26.05.2007, 04.06.2007, 29.05.2010, 09.06.2014, 03.06.2016, 07.06.2016, 25.06.2017, 06.07.2017, 07.07.2019, 02.07.2020, 03.07.2020 — 1-10 sp. per day.

\section{Pedias ia contaminella (Hübner, 1796)}

M a t e r i a l . D: 19.06.2016, 24.06.2016, 27.06.2016, 27.06.2017, 28.07.2017, 17.08.2018, 31.08.2018, 14.09.2018, 07.06.2019, 21.06.2019, 20.07.2019, 03.08.2019, 09.08.2019, 16.08.2019, 06.09.2019, 19.06.2020, 26.06.2020, 14.08.2020, 28.08.2020, 18.09.2020$1-2$ sp. per night.

\section{Pediasia aridella (Thunberg, 1794)}

M a t e ri a l. D: $14.09 .2018-1$ sp.

Pediasia matricella (Treitschke, 1832)

M a t e ri a l . D: 04.09 .2015 - 1 sp., 18.09 .2015 - 2 sp. P: 08.09 .2018 - 1 sp., 08.09 .2019 - 2 sp., 30.08 .2020 - 1 sp., 13.09.2020 - $10 \mathrm{sp}$.

\section{Platytes cerus se Ila ([Denis et Schiffermüller], 1775)}

M a t e ri a l. D: 12.05.2012, 28.05.2012, 16.05.2014, 24.05.2014, 30.05.2015, 12.06.2015, 19.06.2015, 27.05.2016, 03.06.2016, 05.06.2016, 23.06.2017, 18.05.2018, 09.06.2018, 24.05.2019, 23.05.2020, 31.05.2019, 07.06.2019, 13.06.2020, 19.06.2020 - 1-10 sp. per night. L: 23.05.2016 - 1 sp., 03.06.2016 - tens of $\hat{\delta}$ and 2 + + , 07.06.2016 - 3 sp., 24.05.2017 - 2 sp., 27.05.2017 - 3 sp., 01.06.2017 - 3 sp., 03.06.2018 — tens, 04.06.2018 — tens, 06.06.2018 — tens, 06.06.2019 - tens.

\section{Platytes alpinella (Hübner, [1813])}

M a t e r i a l . D: 07.08.2013, 24.07.2015, 07.08.2015, 14.08.2015, 15.07.2015, 17.07.2015, 26.07.2015, 31.07.2015, 19.08.2016, 27.08.2016, 28.07.2017, 04.08.2017, 19.08.2017, 03.08.2018, 17.08.2018, 16.08.2019, 29.08.2020 - 1-10 sp. per night. P: 13.08.2005, 04.08.2006, 19.07.2015, 02.08.2015, 29.08.2015, 06.07.2017, 12.08.2018, 26.08.2018, 18.08.2019, 01.08.2020, 16.08.2020, 23.08.2020 1-10 sp. per day.

Ta lis que rce lla ([Denis et Schiffermüller], 1775)

M a teri a l. D: $14.08 .2013-1$ sp. C: $13.08 .2013-1$ sp.

\section{Schoenobius gigantella ([Denis et Schiffermüller], 1775)}

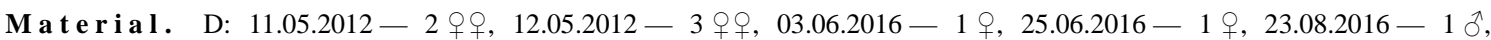
07.06.2019-1 + .

\section{Donacaula forficella (Thunberg, 1794)}

M a te ri a l. D: $03.07 .2016-1$ sp.

Scirpophaga praelata (Scopoli, 1763)

M a teri a l. D: $24.06 .2016-1$ + .

\section{Elophila nymphaeata (Linnaeus, 1758)}

M a t e r i a l. D: 01.09.2007, 29.07.2008, 06.08.2008, 28.05.2012, 27.07.2012, 14.08.2013, 10.07.2015, 17.07.2015, 31.07.2015, 27.06.2017, 17.08.2018, 14.09.2018, 18.05.2019, 31.05.2019, 09.08.2019 - 1-2 sp. per night. L: 27.05.2000, 26.05.2002, 26.08.2003, 07.08.2005, 27.05.2006, 01.06.2006, 04.06.2007 - 1-3 sp. per day.

\section{Acentria ephemerella ([Denis et Schiffermüller], 1775)}

M a t e ri a l. D: 24.05.2014 - 1 sp., 12.06.2015 - 2 sp., 28.06.2015 - 1 sp., 31.07.2015 - 2 sp., 04.09.2015 - 1 sp., 03.06.2016 - 1 sp., 19.06.2016 - 6 sp., 24.06.2016 - 1 sp., 28.07.2017 - 5 sp., 04.08.2017 - 1 sp., 07.06.2020 - 1 sp.

\section{Catac lysta le m nata (Linnaeus, 1758)}

M a t e r i a l . D: 23.07.2008, 20.08.2008, 12.05.2012, 14.06.2012, 07.08.2013, 16.05.2014, 24.05.2014, 12.06.2015, 04.09.2015, 19.06.2016, 25.06.2016, 17.07.2016, 26.07.2016, 27.06.2017, 09.06.2018, 06.07.2018, 14.09.2018, 27.06.2019, 07.06.2020, 12.06.2020, 13.06.2020, 26.06.2020, 03.07.2020, 18.07.2020, 31.07.2020, 29.08.2020 - 1-4 sp. per night. L: 27.05.2000, 12.06.2001, 14.08.2001, 15.08.2001, 23.08.2001, 17.05.2004, 23.05.2005, 07.08.2005, 13.08.2005, 18.05.2006, 23.05.2006, 30.05.2006, 01.06.2006, 03.06.2006, 04.06.2006, 06.06.2006, 12.06.2006, 01.07.2006, 15.07.2006, 01.08.2006, 03.08.2006, 04.08.2006, 15.08.2006, 24.05.2007, 26.05.2007, 29.05.2007, 30.06.2007, 17.05.2014 - 1-20 sp., near water.

\section{Parapoynx stratiotata (Linnaeus, 1758)}

M a t e r i a l . D: $07.07 .2007,23.07 .2008,06.08 .2008,20.08 .2008,06.09 .2008,19.05 .2012,22.06 .2012,10.08 .2012,24.05 .2014$, 30.05.2015, 26.06.2015, 28.06.2015, 03.07.2015, 10.07.2015, 17.07.2015, 24.07.2015, 31.07.2015, 14.08.2015, 04.09.2015, 27.05.2016, 03.06.2016, 03.07.2016, 06.08.2016, 27.08.2016, 26.05.2017, 28.07.2017, 19.08.2017, 25.05.2018, 27.07.2018, 03.08.2018, 17.08.2018, 31.08.2018, 24.05.2019, 09.08.2019, 07.06.2020, 12.06.2020, 08.08.2020 - 1-10 sp. per night. 
Aporodes floralis (Hübner, [1809])

M a t e r i a l . D: 14.08.2013, 26.06.2015, 29.08.2015, 27.06.2017, 27.07.2018, 17.08.2018 — 1 sp. per night. C: 30.09 .2015 1 sp.

Cynaeda dentalis ([Denis et Schiffermüller], 1775)

M a t e r i a l . D: $\quad$ 28.06.2015 - 1 sp., 03.07.2015 - 1 sp., 10.07.2015 - 1 sp., 04.09.2015 - 1 sp., $12.09 .2015-2$ sp., 08.09.2018 - 1 sp., 14.09.2019 - 1 sp. C: $09.08 .2000,17.08 .2002,13.09 .2005,11.06 .2011,13.07 .2015,02.08 .2015,23.08 .2016$, 26.08.2018 - 1 sp. per night.

\section{Epascestria pustulalis (Hübner, [1823])}

M a t e r i a l . P: 25.06.2017 - 5 sp., 08.06.2018 - 1 sp., 28.07.2018 - 1 sp., 02.07.2020 - 1 sp.

Atralata albofascialis (Treitschke, 1829)

M a t e r i a l . P: 09.05.2016 - 1 sp. near aspen-birch forest.

Evergestis frumentalis (Linnaeus, [1760])

M a t e r i a l . D: 12.05.2012, 19.05.2012, 24.05.2014, 30.05.2015, 13.05.2016, 27.05.2016, 05.06.2016, 26.05.2017, 04.05.2018, 09.05.2018, 18.05.2018, 18.05.2019, 01.05.2020, 12.06.2020 - 1-2 sp. per night. C: 16.05 .2010 - 1 sp., 08.05 .2012 - 1 sp., $14.05 .2017-$ 1 sp. P: 17.06.2001, 25.05.2003, 07.05.2004, 17.05.2004, 15.06.2004, 26.05.2005, 27.05.2006, 13.05.2007, 20.05.2007, 22.05.2015, 11.06.2015, 15.05.2016, 11.05.2019, 30.05.2020, 07.06.2020 - 1-8 sp. per day.

\section{Evergestis forficalis (Linnaeus, 1758)}

M a t e r i a l. D: 22.05.2010 - 1 sp., 12.05.2012 - 1 sp., 03.05.2014 - 1 sp., 24.05.2019 - 1 sp., $09.08 .2019-1$ sp.

\section{Evergestis extimalis (Scopoli, 1763)}

M a t e r i a l . D: 27.05 .2016 - 1 sp., 28.07.2017 - 1 sp., 09.06.2018 - 1 sp. C: $30.05 .2000-1$ sp., $03.06 .2000-1$ sp., 05.06.2005 - 1 sp., 24.08.2005 - 1 sp., 02.07.2007 - 1 sp. L and P: 15.06.2000, 23.05.2005, 25.05.2005, 31.05.2005, 11.07.2005, 03.06.2006, 06.06.2006, 12.06.2006, 04.08.2006, 24.05.2007, 04.06.2007, 06.06.2007 - 1-2 sp. per day.

\section{Evergestis pallidata (Hufnagel, 1767)}

M a t e r i a l . D: 07.08 .2013 - 1 sp., 16.05.2014 - 1 sp., 12.06.2015 - 1 sp., 07.08 .2015 - 1 sp., 27.05 .2016 - 1 sp., 03.07.2016 — 1 sp. L: 04.06 .2007 — 1 sp. P: 27.08 .2003 — 1 sp.

\section{Evergestis politalis ([Denis et Schiffermüller], 1775)}

M a t e r i a l . D: 12.05 .2012 - 1 sp. P: 25.05.2000, 16.05.2002, 17.05.2002, 17.05.2015, 22.05.2015, 28.05.2016, 02.09.2018, 16.05.2020 - 1-2 sp. per day.

\section{* Hellula undalis (Fabricius, 1781)}

M a t e ri a l. D: $14.09 .2018-1$ sp.

Loxostege turbidalis (Treitschke, 1829)

M a t e ri a l. P: 31.05.2020 - 1 sp., 19.07.2020 - 1 sp.

\section{L o x o stege de liblatica Szent-Ivány et Uhrik-Mészáros, 1942}

M a t e ria l. D: 28.05 .2012 - 1 sp., 14.08 .2015 - 1 sp., 03.06.2016 - 1 sp. P: 29.05.2000, 13.05.2002, 16.05.2002, 17.05.2002, 27.05.2002, 17.05.2003, 16.06.2004, 16.05.2005, 19.05.2005, 23.05.2005, 11.07.2005, 27.05.2006, 01.06.2006, 04.08.2006, 20.05.2007, 22.05.2007, 23.05.2007, 21.05.2008, 10.05.2010, 16.05.2010, 29.05.2010, 22.06.2014, 19.07.2015, 26.07.2015, 02.08.2015, 09.08.2015, 14.05.2017, 28.05.2017, 09.05.2018, 27.05.2018, 13.07.2019, 17.05.2020, 02.07.2020, 12.07.2020, 18.07.2020 - 1-10 sp. per day.

\section{Loxostege sticticalis (Linnaeus, [1760])}

M a t e r i a l. D: $12.09 .2011-3$ sp., 10.09.2011 - 10 sp., 19.05.2012 - 1 sp., $15.09 .2012-1$ sp., $24.05 .2014-1$ sp., 14.08.2015 - 2 sp., 19.08.2017 - 1 sp. L and P: 20.05.2000, 23.05.2000, 29.05.2000, 04.06.2000, 05.06.2000, 06.06.2000, 10.06.2000, 23.07.2000, 11.06.2001, 17.06.2001, 21.06.2001, 25.06.2001, 01.07.2001, 05.07.2001, 28.07.2001, 06.08.2001, 14.08.2001, 15.08.2001, 10.05.2002, 12.05.2002, 27.05.2002, 12.08.2002, 01.09.2002, 24.08.2003, 27.08.2003, 17.05.2004, 23.05.2005, 25.05.2005, 30.05.2005, 31.05.2005, 13.08.2005, 21.08.2005, 26.05.2006, 30.05.2006, 01.06.2006, 03.08.2006, 04.08.2006, 13.08.2006, 15.08.2006, 22.05.2007, 24.05.2007, 04.06.2007, 06.06.2007, 11.06.2011, 27.07.2011, 14.08.2011, 11.09.2011, 06.10.2011, 15.09.2013, 10.05.2014, 17-18.05.2014, 24.05.2015, 06.09.2015 - 1-100 sp. per day.

\section{Ecpyrrhorrhoe rubiginalis (Hübner, 1796)}

M a t e r i a l . D: $\quad$ 07.08.2013, 10.07.2015, 27.06.2016, 06.08.2016, 23.06.2017，27.06.2017, 21.06.2019, 16.08.2019, 28.06.2020 — 1 sp. per night. L: 05.05.2005 — 1 sp., 05.05.2012 — 1 sp.

\section{Paracorsia repandalis ([Denis et Schiffermüller], 1775)}

M a t e r i a l . D: 12.05.2012, 07.08.2013, 14.08.2013, 10.05.2014, 24.05.2014, 03.07.2015, 07.08.2015, 14.08.2015, 04.09.2015, 13.05.2016, 27.06.2016, 01.07.2016, 03.07.2016, 06.08.2016, 23.08.2016, 23.06.2017, 09.05.2018, 27.07.2018, 03.08.2018, 17.08.2018, 24.05.2019, 09.08.2019, 16.08.2019, 24.07.2020, 31.07.2020, 08.08.2020, 14.08.2020, 21.08.2020, 28.08.2020, 29.08.2020 - 1-5 sp. per night. C: 18.08 .2011 - 1 sp., 02.08 .2015 - 1 sp., 11.08.2015 - 2 sp., 03.09 .2015 - 1 sp., 17.08 .2017 - 1 sp., $26.08 .2018-1$ sp. 
P: 13.08.2006 — 1 sp., 10.05.2010 - 1 sp., 21.07.2015 - 2 larvae and 1 pupa on Verbascum densiflorum, 09.05.2016 - 1 sp. L: $16.05 .2005-1$ sp.

* Paratalanta pandalis (Hübner, [1825])

M a t e ri a l. L: $16.05 .2007-1$ sp.

Paratalanta hyalinalis (Hübner, 1796)

M a t e r i a l . D: 03.07.2015 — 1 sp., 24.06.2016 — 1 sp., 05.07.2019 — 1 sp. L: 22.06.2019 — 1 sp., 03.07 .2020 - 1 sp.

Pyrausta sanguinalis (Linnaeus, 1767)

M a t e r i a l . D: 11.08.2007, 12.05.2012, 16.05.2014, 30.05.2015, 03.07.2015, 10.07.2015, 24.07.2015, 07.08.2015, 14.08.2015, 13.05.2016, 27.05.2016, 05.06.2016, 27.06.2016, 01.07.2016, 26.07.2016, 06.08.2016, 13.08.2016, 26.05.2017, 28.07.2017, 19.08.2017, 04.05.2018, 09.05.2018, 06.07.2018, 27.07.2018, 03.08.2018, 18.05.2019, 01.05.2020, 31.07.2020, 08.08.2020 - 1-4 sp. per night. P: 10.05.2004, 23.05.2008, 22.05.2008, 09.05.2016, 13.05.2016, 15.05.2016, 27.06.2016, 27.05.2018, 05.08.2018, 19.07.2020, 01.08.2020, 02.08.2020, 23.08.2020 — 1-20 sp., mostly on Thymus pallasianus. L: $17.05 .2014-1$ sp., $18.05 .2014-1$ sp., $14.05 .2017-1$ sp.

\section{Pyrausta despicata (Scopoli, 1763)}

M a t e r i a l . D: 23.07.2008, 28.04.2013, 13.09.2013, 31.07.2015, 07.08.2015, 14.08.2015, 04.09.2015, 18.09.2015, 24.06.2016, 23.06.2017, 27.06.2017, 28.07.2017, 04.08.2017, 19.08.2017, 30.04.2018, 27.07.2018, 03.08.2018, 31.08.2018, 20.07.2019, 24.07.2020, 14.08.2020, 21.08.2020 — 1-4 sp. per night. L and P: 23.04.2000, 20.05.2000, 01.07.2001, 14.08.2001, 29.04.2002, 04.07.2002, 26.08.2002, 02.05.2004, 04.05.2004, 05.05.2004, 05.05.2005, 18.05.2011, 24.04.2016, 02.08.2020 - 1-10 sp. per day.

\section{Pyrausta aurata (Scopoli, 1763)}

M a t e r i a l . D: 12.07.2007, 16.08.2007, 23.07.2008, 17.05.2011, 07.08.2013, 03.07.2015, 10.07.2015, 17.07.2015, 31.07.2015, 07.08.2015, 14.08.2015, 04.09.2015, 27.06.2016, 01.07.2016, 03.07.2016, 15.07.2016, 26.07.2016, 13.08.2016, 19.08.2016, 23.08.2016, 27.08.2016, 19.09.2017, 25.05.2018, 09.06.2018, 17.08.2018, 21.06.2019, 27.06.2019, 26.06.2020, 03.07.2020, 21.08.2020, 28.08.2020 1-5 sp. per night. L: 30.07.2006 — 1 sp., 18.05.2011 — 1 sp., 14.05.2017 — 1 sp., 20.07.2020 — 1 sp.

Pyrausta purpuralis (Linnaeus, 1758)

M a t e r i a l. D: 31.07.2015 — 1 sp., 14.08.2015 - 1 sp., 24.06.2016 — 1 sp. L: 05.05 .2004 — 1 sp. P: 05.05 .2005 — 1 sp.

Pyrausta rectefas cialis (Toll, 1936) sensu Slamka, 2013

M a te ri a l. D: $27.07 .2007-1$ sp.

Uresiphita gilvata (Fabricius, 1794)

M a t e ri a l . D: 24.05 .2019 - 1 sp. P: 28.05 .2017 - 5 sp., 21.06.2020 - 10 larvae, 26.06 .2020 - 6 larvae, 02.08 .2020 10 larvae, on Genista tinctoria.

Nas c ia cilialis (Hübner, 1796)

M a t e r i a l. D: 19.05 .2012 - 1 sp., 18.05.2018 - 1 sp., 24.05.2019 - 1 sp.

Sitochroa palealis ([Denis et Schiffermüller], 1775)

M a t e ri a l. D: $19.06 .2016-1$ sp. L: $30.07 .2006-1$ sp., $03.08 .2006-1$ sp., $18.07 .2020-1$ sp. P: $11.06 .2016-1$ sp., 20.07.2020-1 sp.

\section{Sitochroa verticalis (Linnaeus, 1758)}

M a t e r i a l. D: 07.07.2007, 12.05.2012, 30.05.2015, 12.06.2015, 31.07.2015, 14.08.2015, 03.06.2016, 27.06.2016, 15.07.2016, 17.07.2016, 06.08.2016, 26.05.2017, 23.06.2017, 27.06.2017, 28.07.2017, 19.08.2017, 18.05.2018, 25.05.2018, 02.06.2018, 09.06.2018, 18.05.2019, 03.08.2019, 16.08.2019, 13.06.2020, 08.08.2020 - 1-3 sp. per night. C: 28.06.2008 - 1 sp., 07.05.2010 — 1 sp. L: 06.06.2006, 01.07.2006, 15.07.2006, 29.05.2007, 31.05.2007, 06.06.2007, 14.06.2007, 30.06.2007, 14.08.2011, 23.08.2015, 23.05.2020, 03.07.2020 1-5 sp. per day. P: 27.05.2006, 1205.2007, 22.05.2007, 26.05.2007, 01.07.2017, 06.07.2017, 30.05.2020, 07.06.2020, 26.06.2020, 08.08.2020 - 1-10 sp. per day.

\section{Sclerocona acutellus (Eversmann, 1842)}

M a t e r i a l. D: $14.06 .2012-1$ sp., 12.06.2015 - 2 sp., 19.06.2016 - 1 sp., 24.06.2016 - 1 sp., 25.06.2016 - 1 sp., 27.06.2016 — 2 sp., 23.06.2017 - 1 sp., 27.06.2017 — 5 sp., 07.06.2019 - 1 sp., 21.06.2019 — 1 sp., 12.06 .2020 - 1 sp., $26.06 .2020-$ 1 sp., $03.07 .2020-1$ sp.

\section{Ostrinia nubilalis (Hübner, 1796)}

M a terial. P: $25.05 .2005-1 \delta$.

Ostrinia scapulalis (Walker, 1859)

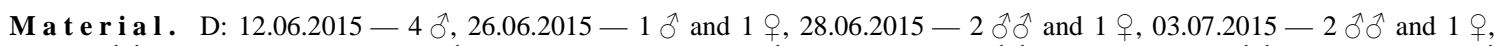

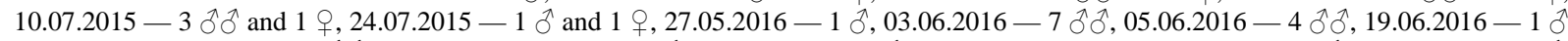

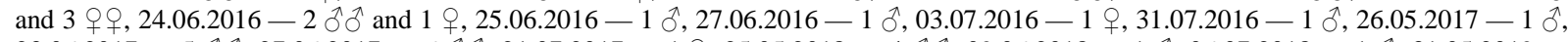

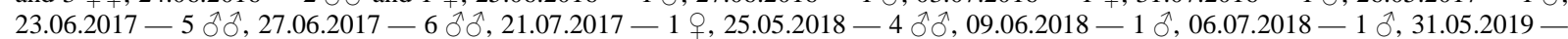

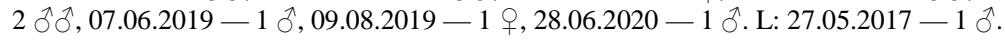




\section{Anania verbascalis ([Denis et Schiffermüller], 1775)}

M a t e ri a l . $\quad$ D: 19.05 .2012 - 1 sp., 17.07.2016 - 1 sp., 19.08.2017 - 1 sp. L and P: 27.05.2000, 16.05.2004, 25.05.2005, 31.05.2005, 06.08.2005, 13.08.2005, 12.06.2006, 15.07.2006, 03.08.2006, 15.08.2006, 22.05.2007, 24.05.2007, 26.05.2007, 29.05.2007, 04.06.2007, 21.05.2008, 11.06.2015, 24.05.2017 — 1 sp. per day.

\section{Anania coronata (Hufnagel, 1767)}

M a t e r i a l . D: 19.06.2016 — 1 sp., 12.06.2020 — 1 sp. L: 16.05.2007 — 1 sp., 24.05.2017 — 1 sp.

Anania perlucidalis (Hübner, [1809])

M a t e r i a l . D: 03.06.2016 - 1 sp., 21.06.2019 - 1 sp.

Anania terrealis (Treitschke, 1829)

M a t e r i a l . D: $12.05 .2012,19.05 .2012,10.07 .2015,05.06 .2016,06.08 .2016,28.07 .2017,09.05 .2018,18.05 .2018,25.05 .2018$, 06.07.2018, 31.08.2018, 18.05.2019, 31.05.2019, 18.07.2020, 21.08.2020, 29.08.2020 - 1-3 sp. per night. C: 31.05.2015 - 1 dead sp., 05.06.2015 - 1 sp., 29.08 .2018 - 1 sp. P: 17.05.2002, 27.05.2002, 16.05.2004, 04.06.2004, 22.05.2007, 23.05.2007, 21.05.2008, 26.07.2015, 09.05.2016, 14.05.2017, 28.05.2017, 23.07.2017, 15.09.2017, 26.08.2018, 24.05.2020, 30.05.2020 — 1-5 sp. per day.

\section{Anania hortulata (Linnaeus, 1758)}

M a t e r i a l . D: 07.06.2008 — 1 sp., 28.05.2012 — 1 sp., 03.07.2015 — 1 sp., 09.06.2018 — 1 sp. L: 12.06.2001, 23.05.2005, 27.05.2006, 04.06.2006, 12.06.2006, 15.07.2006, 04.06.2007, 17.06.2007, 30.06.2007, 05.07.2007 — 1-2 sp. per day. P: 01.06.2006 — 1 sp., 31.05.2020 - $1 \mathrm{sp}$

\section{Udea ferrugalis (Hübner, 1796)}

M a t e r i a l . D: 13.08.2016 - 2 sp., 23.08.2016 - 1 sp., 29.08.2020 - 1 sp., $16.10 .2020-1$ sp.

\section{Udea accolalis (Zeller, 1867)}

M a t e r i a l . D: 17.07 .2015 - 1 sp., 07.08.2015 - 1 sp., 26.05.2017 - 3 sp., 18.05.2019 - 1 sp. L: $16.05 .2005-1$ sp., 15.05.2011 - 1 sp., 18.05.2011 - 2 sp., 14.05.2017 - 2 sp. P: 13.05.2007 - 3 sp., 18.05.2008 - 3 sp., 02.05.2017 - 1 q coll.

\section{Patania ruralis (Scopoli, 1763)}

M a t e r i a l. D: $07.07 .2007,23.07 .2008,26.08 .2011,28.05 .2012,04.06 .2012,14.06 .2012,22.06 .2012,09.08 .2014,07.08 .2015$ 29.08.2015, 04.09.2015, 27.08.2016, 27.06.2017, 28.07.2017, 04.08.2017, 09.09.2017, 17.08.2018, 31.08.2018, 08.09.2018, 07.06.2019, 06.09.2019, 14.09.2019, 26.06.2020, 28.08.2020 - 1-6 sp. per night. L: 10.09.2005, 30.05.2006, 01.07.2006, 15.07.2006, 15.08.2006, 20.06.2007 - 1-2 sp. per day.

\section{Mecyna flavalis ([Denis et Schiffermüller], 1775)}

M a t e r i a l . L: 06.09.2015 - 10 sp., 13.09.2015 - 1 sp., 26.09.2015 - 1 sp., 03.06.2016 - 100 sp., 07.06 .2016 - 50 sp., 03.06.2018 - 15 sp., 04.06.2018 — 2 sp., 06.06.2018 - 2 sp., 10.07.2020 - 6 sp., 09.08.2020 — 3 sp.

Dolicharthria punctalis ([Denis et Schiffermüller], 1775)

M a te ri a l. D: $16.08 .2019-1$ o . C: $04.08 .2020-1$ sp.

\section{Nom ophila noctuella ([Denis et Schiffermüller], 1775)}

M a t e r i a l . D: 07.07.2007, 12.07.2007, 27.07.2007, 28.07.2007, 11.08.2007, 01.09.2007, 17.05.2011, 26.08.2011, 12.05.2012, 19.05.2012, 28.05.2012, 10.05.2014, 19.06.2015, 26.06.2015, 28.06.2015, 03.07.2015, 10.07.2015, 17.07.2015, 24.07.2015, 31.07.2015, 07.08.2015, 14.08.2015, 04.09.2015, 18.09.2015, 25.09.2015, 02.10.2015, 03.10.2015, 03.06.2016, 19.06.2016, 24.06.2016, 25.06.2016, 01.07.2016, 03.07.2016, 08.07.2016, 26.07.2016, 31.07.2016, 06.08.2016, 13.08.2016, 19.08.2016, 23.08.2016, 27.08.2016, 09.09.2016, 28.07.2017, 03.08.2018, 17.08.2018, 31.08.2018, 18.05.2019, 07.06.2019, 21.06.2019, 27.06.2019, 05.07.2019, 20.07.2019, 03.08.2019, 16.08.2019, 19.06.2020, 03.07.2020, 18.07.2020, 24.07.2020, 31.07.2020, 08.08.2020, 14.08.2020, $16.10 .2020-1-25$ sp. per night. C: 03.06.2000, 08.06.2000, 31.08.2003, 11.10.2003, 28.10.2006, 22.09.2007, 19.09.2011, 14.05.2014, 16.08.2014, 18.07.2015, 19.07.2015, 14.04.2016 - 1-3 sp. per night. L: 15.06.2000, 19.04.2001, 27.04.2001, 01.07.2001, 28.07.2001, 06.08.2001, 14.08.2001, 15.08.2001, 23.08.2001, 30.08.2001, 17.08.2002, 06.08.2005, 01.06.2006, 06.06.2006, 15.08.2006, 24.05.2007, 26.05.2007, 17.06.2007, 18.06.2007, 20.06.2007, 14.04.2012, 01.05.2014, 17-18.05.2014, 13.04.2016, 17.04.2016 - 1-3 sp. per day. P: 20.05.2007, 22.05.2007, 24.10.2009, 19.07.2015, 21.07.2015, 26.09.2015, 03.05.2016, 02.09.2019, 23.08.2020 — 1-5 sp. per day.

\section{S u perfa mi ly D R E PA N O I D A Boisduval, 1828}

\section{F a mily D R E PA N I A E Boisduval, 1828}

\section{Thyatira batis (Linnaeus, 1758)}

M a t e r i a l . $\quad$ D: 12.07.2007 - 1 sp., 15.07.2007 - 3 sp., 20.07.2007 - 2 sp., 21.07.2007 - 1 sp., $23.07 .2008-2$ sp., 12.05.2012 — 1 sp., 19.05.2012 — 1 sp., 17.07.2015 — 1 sp., 27.05.2016 — 1 sp., 17.07.2016 — 1 sp., 26.05 .2017 — 1 sp. L: 19.05 .2001 $1 \mathrm{sp}$.

\section{Tethea ocularis (Linnaeus, 1767)}

M a t e ri a l . D: $19.05 .2012-1$ sp., $12.05 .2019-1$ sp. on wine lure, $18.05 .2019-1$ sp. on wine lure, $09.08 .2019-1$ sp. C: $07.08 .2008-1$ dead sp., $19.05 .2010-1$ sp. 
S. O. DEMYANENKO, O. V. BIDZILYA, E. A. KAROLINSKIY New records of Lepidoptera (Insecta) of Severodonetsk (Luhansk Region, Ukraine) and its environs

* Achlya flavicornis (Linnaeus, 1758) on Betula.

M a t e r i a l . D: 22.03.2014 - 2 sp. P: 15.04.2012 - 2 sp., birch forest, 09.05.2018 - 1 larva on Betula, 27.05.2018 - 2 larvae

Watsonalla binaria (Hufnagel, 1767)

M a t e r i a l. D: 23.05.2009 — 1 sp. L: 01.05.2014 — 1 sp. P: 16.09.2018 - 1 larva on Quercus robur.

Cilix glaucata (Scopoli, 1763)

M a t e r i a l. $\quad$ D: 23.05 .2015 - 1 sp., 03.07.2015 - 1 sp., 07.08.2015 - 1 sp., $14.08 .2015-1$ sp., $29.08 .2015-1$ sp., 13.05.2016 - 1 sp., 25.06.2016 - 3 sp., 27.06.2016 - 1 sp., 01.07.2016 - 1 sp., 27.06.2017 — 1 sp., 04.05 .2018 - 1 sp., $09.05 .2018-$ 1 sp., 08.08 .2020 - 2 sp. C: $31.08 .2015-1$ sp.

S uperfamily LA S I C AMP O I E A Harris, 1841

F a mily L A S I O C A P I D A E Harris, 1841

Malacosoma castrense (Linnaeus, 1758)

M a t e ri a l. D: $26.06 .2015-1$ sp.

L a s i o c a mpa trifol i ([Denis et Schiffermüller], 1775)

M a t e r i a l. D: $19.08 .2016-1$ sp., 31.08.2018 - 2 sp., 21.08.2020 - 2 sp., 28.08.2020 - 1 sp. P: 05.06.2016, 28.05.2018, 08.06.2018 and 06.06.2019 - 4 larvae.

Las i c с т pa quercus (Linnaeus, 1758)

M a t e r i a l . P: 07.05.2017, 15.10.2017, 27.04.2018, 14.04.2019 and 04.05.2019 — 5 larvae, birch forests.

Superfamily GEOMETRO IDEA Leach, 1815

Family GEOMETRIDAE Leach, 1815

I daea sylvestraria (Hübner, [1799])

M a t e r i a l . D: 14.08.2013, 12.06.2015, 03.07.2015, 07.08.2015, 14.08.2015, 12.09.2015, 03.06.2016, 05.06.2016, 23.08.2016, 09.06.2018, 31.05.2019, 21.06.2019, 16.08.2019, 15.08.2020, 29.08.2020 - 1-5 sp. per night. P: 11.06.2015 — 1 sp., 28.05.2016 - 1 sp.

Idaea mancipiata (Staudinger, 1871)

M a t e r i a l . D: $15.08 .2013-1$ sp., 19.06.2016 - 1 sp.

* Idaea trigeminata (Haworth, [1809])

M a te ri a l. D: $24.06 .2018-1$ sp.

I da e a de ver s aria (Herrich-Schäffer, 1847)

M a t e r i a l . D: $21.08 .2020-1$ sp. P: $25.06 .2017-2$ sp., 08.06.2019 - 1 ô coll.

S copula ornata (Scopoli, 1763)

M a t e r i a l . D., 19.08.2016 - 1 sp., 03.08.2019 - 1 sp., 28.08.2020 - 1 sp. P: 18.07 .2020 - 1 sp.

S c opula ochraceata (Staudinger, 1901)

M a t e ri a l . D: 04.09 .2015 - 2 sp., 19.08.2016 - 1 sp., 17.08 .2018 - 1 sp., 08.09 .2018 - 1 sp. P: $03.09 .2016-1$ sp., 11.09.2016 - 5 sp., 28.05.2017 - 2 sp., 31.05.2020 - 1 sp., 13.09.2020 - 2 sp.

S c ор u la subpunctaria (Herrich-Schäffer, 1847)

M a t e ri a l . D: 03.07.2015 - 1 sp., 25.06.2016 - 1 sp., 27.06.2016 - 2 sp., 01.07.2016 - 2 sp., 27.06.2017 - 1 sp. C: 12.07.2017 — 1 sp. L: 02.07.2016 — 1 sp. P: 03.07.2020 — 1 sp. in deciduous forest.

Cyclophora porata (Linnaeus, 1767)

M a terial. D: $14.06 .2012-1$ sp.

Catac lys me riguata (Hübner, [1813])

M a t e ri a l. D: $24.05 .2019-1$ sp.

* Scotopteryx mucronata (Scopoli, 1763)

M a t e r i a l. $04.06 .1990-1$ , Severov I.G., det. Kostijuk I.Yu., $06.1991-1$ ô, Severov I.G., det. Kostijuk I.Yu. P: 28.05.2018 - 2 के ồ coll.

Catarhoe rubidata ([Denis et Schiffermüller], 1775)

M a t e ri a l. D: 20.07.2019-1 sp. 
Xanthorhoe biriviata (Borkhausen, 1794)

M a teria l. D: $24.06 .2016-1$ sp.

Anticlea derivata ([Denis et Schiffermüller], 1775)

M a t e ri a l . D: 0.04.2018 - 1 sp., 04.05.2018 - 1 sp. P: 06.05.2018 - 1 sp., aspen-birch forest.

* Chloroclysta siterata (Hufnagel, 1767)

M a te ri a l. P: $12.09 .2017-1$ sp.

* Minoa murinata (Scopoli, 1763)

M a t e r i a l . P: 28.05 .2016 - 10 sp., 05.06.2016 - 1 sp., 28.05.2017 - 3 sp., $01.06 .2019-1$ sp., $24.05 .2020-1$ sp., 24.05.2020 - 1 sp., meadows in birch forests, near Euphorbia $\left(48.9180^{\circ} \mathrm{N}, 38.6405^{\circ} \mathrm{E}\right.$ and $\left.48.9634^{\circ} \mathrm{N}, 38.6045^{\circ} \mathrm{E}\right)$.

* Hydria cervinalis (Scopoli, 1763)

M a teria l. D: $04.05 .2018-1 \mathrm{sp}$.

Horis me vitalbata ([Denis et Schiffermüller], 1775)

M a te ri a l. P: $15.05 .2016-1$ sp.

* Horisme tersata ([Denis et Schiffermüller], 1775)

M a te ri a l. D: $27.05 .2016-1$ sp.

* Anticollix sparsata (Treitschke, 1828)

M a t e ri a l. D: $30.06 .2019-1$ \%.

Perizoma alchemillata (Linnaeus, 1758)

M a t e r i a l . 10.07.1990 - 1 sp. (Severov I.G.).

Perizoma lugdunaria (Herrich-Schäffer, [1855])

M a t e ri a l. D: 17.07 .2016 - 1 sp., 18.07.2020 - 1 sp.

Perizoma bifaciata (Haworth, [1809])

M a t e ri a l. D: $16.08 .2019-1$ sp.

* Eup ithec ia pygmaeata (Hübner, [1799])

M a t e r i a l . P: 11.05.2020 - 2 sp., 24.05.2020 - 1 sp., 07.06.2020 - 2 sp., meadows in birch forests, on Stellaria.

* E up ithecia dodone a ta Guenée, [1858]

M a te ria l. D: $26.05 .2017-1$ sp.

E upithe cia simpliciata (Haworth, [1809])

M a t e ri a l. D: $31.07 .2015-1$ sp., 08.08.2020 — 1 sp. L: 24.08 .2020 - 1 sp.

Eup ithe cia in notata (Hufnagel, 1767)

M a te ri a l. D: 09.05.2018-1 1 .

E up ithe c i a o chridata Schütze et Pinker, 1968

M a teria l. D: 09.05.2018-1 , $14.09 .2018-1$ q.

E up ith e cia indigata (Hübner, [1813])

M a t e ri a l. D: 28.04.2013-2 sp., 09.05.2018 - 1 sp.

E upithe cia centaureata ([Denis et Schiffermüller], 1775)

M a t e r i a l . D: 11.05.2013, 12.09.2015, 03.06.2016, 06.08.2016, 25.05.2018, 12.06.2020, 08.08.2020, 15.08.2020 - 1 sp. per night. C: 11.08 .2020 — 1 sp. L: $11.05 .2016-1$ sp.

E up ithecia as s imilata Doubleday, 1856

M a t e ri a l. D: $09.05 .2018-1$ \% .

Eupithecia vulgata (Haworth, [1809])

M a terial. D: $18.05 .2018-1$ §.

Eupithecia millefoliata (Rössler, 1866)

M a t e ri a l. D: $10.07 .2015-1$ sp.

Archiearis parthenias (Linnaeus, [1760])

M a t e r i a l. P: 10.04.2010, 02.04.2011, 24.04.2011, 15.04.2012, 23.03.2014 and 12.04.2015 - 1-20 sp. per day, birch forests, 28.05.2018 - 1 larva, birch forest. 
S. O. DEMYANENKO, O. V. BIDZILYA, E. A. KAROLINSKIY New records of Lepidoptera (Insecta) of Severodonetsk (Luhansk Region, Ukraine) and its environs

Geometra papilionaria Linnaeus, 1758

M a t e r i a l. P: 28.05.2016 - 1 sp., aspen-birch forest.

En nomos fuscantaria (Haworth, [1809])

M a t e ri a l. D: $11.09 .2020-1$ sp. P: 13.07.2019 - 1 pupa between fastened leaves of Alnus glutinosa, imago hatched on 23.07.2019.

Petrophora chlorosata (Scopoli, 1763)

M a t e r i a l. P: 28.05.2016 - common, 02.05.2017 - 10 sp., 08.05.2017 - common, 27.04.2018 - common, 06.05.2018 2 sp., 28.05.2018 - 1 sp., 04.05.2019 - 1 sp., alder and birch forests.

Ma caria artesiaria ([Denis et Schiffermüller], 1775)

M a te ria l. D: $01.07 .2016-1$ sp.

Digrammia rippertaria (Duponchel, 1830)

M a t e ri a l. D: $28.06 .2015-1$ sp. P: $09.05 .2016-1$ sp.

Narraga fasciolaria (Hufnagel, 1767)

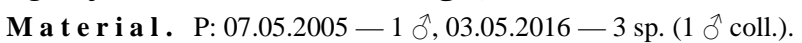

Neognopharmia stevenaria (Boisduval, 1840)

M a t e ri a l. D: $14.06 .2012-1$ sp.

Odontognophos dumetata (Treitschke, 1827)

M a t e ri a l. D: 08.09.2018 - 1 sp., 14.09.2018 - 1 sp.

Charissa obscurata ([Denis et Schiffermüller], 1775)

M a t e r i a l. D: $14.08 .2013-1$ sp., 03.08.2019 - 1 sp., $14.08 .2020-1$ sp., $28.08 .2020-1$ sp.

A p o c he i ma hispidaria ([Denis et Schiffermüller], 1775)

M a t e ri a l. C: 30.03 .2012 — 1 sp., 01.04.2013 — 1 sp. L: 01.05.2014 — 1 larva.

S uperfamily N O C T U O I D E A Latreille, 1809

Family EREBIDAE Leach, 1815

Ei lema pseudocomplana (Daniel, 1939)

M a te ri a l. D: $26.07 .2016-1$ sp.

Family NOCTUID A E Latreille, 1809

Cucullia verbasci (Linnaeus, 1758)

M a t e r i a l . P: 30.05.2020 - 6 larvae of different stages, 07.06.2020 - 1 larva, 10.06.2020 - 1 larva, 12.06.2020 — 1 larva, 19.06.2020 - 1 larva, on Verbascum, mostly V. densiflorum.

A steroscopus sphinx (Hufnagel, 1766)

M a t e ri a l. D: $13.10 .2019-1$ sp.

Athetis gluteosa (Treitschke, 1835)

M a te ri a l. D: $07.06 .2020-1$ sp.

* Ti li a c e a s u lph u rago ([Denis et Schiffermüller], 1775)

M a t e ri a l. D: 28.09.2019 - 1 sp., 11.09.2020 - 1 sp.

Agrochola helvola (Linnaeus, 1758)

M a teri a l. D: $04 \cdot 10.2019-1 \mathrm{sp.}$

Agrochola lota (Clerck, 1759)

M a teri a l. C: $07.10 .2020-1 \mathrm{sp.}$

Antitype chi (Linnaeus, 1758)

M a teri a l. D: $14.09 .2019-1 \mathrm{sp.}$

Rhizedra l utosa (Hübner, [1803])

M a t e ri a l. D: $04.10 .2019-2$ sp., $16.10 .2020-2$ sp. 
Conclusions. According to the results of our research and the literature data, 1,074 species of Lepidoptera are found in Severodonetsk and its environs, 425 of which are given for the first time, 190 of which are new records for Luhansk Region, and one is new for Ukraine. At the same time, the list of Lepidoptera of Severodonetsk is still not complete and further studies will certainly supplement it with new species.

Acknowledgements. The authors are sincerely grateful to Yu. Budashkin (T. I. Vyazemsky Karadag Scientific Station - Nature Reserve, Kurortnoye, Feodosia), Yu. Geryak (Sambir), O. Gorbunov (A. N. Severtsov Institute of Ecology and Evolution of the Russian Academy of Sciences, Moscow, Russia), Yu. Guglya (Museum of Nature of the Vasyl Karazin Kharkiv National University, Kharkiv), K. Efetov (S. I. Georgievskyi Crimean State Medical University, Simferopol), I. Kostjuk (Zoological Museum of the Taras Shevchenko National University of Kyiv), E. Rutjan (I. I. Schmalhausen Institute of Zoology of the National Academy of Sciences of Ukraine, Kyiv), I. Severov (Rubezhnoe), V. Sergienko (Kyiv), V. Yepishin (Institute for Evolutionary Ecology of the National Academy of Sciences of Ukraine, Kyiv) for consultations in some difficult determination cases.

\section{REFER E N C E S}

Bidzilya, A. V. 1994 (1995). New and little-known species of the Microlepidoptera of the fauna of Ukraine [Новые и малоизвестные виды Microlepidoptera фауны Украины]. Journal of the Ukrainian Entomological Society [Журнал Українського ентомологічного товариства], 2(1), 35-38. URL: http://www.lepidoptera.crimea.ua/articles/Bidzilya_1995_Microlepidoptera_Ukraina.djvu. [in Russian].

Bidzilya, O. V., Budashkin, Yu. I. 2017. New records of Lepidoptera from Ukraine and description of a new species of Caloptilia Hübner, 1825 (Lepidoptera, Gracillariidae) from the mountains of Crimea. Nota Lepidopterologica, 40(2), 145-161. DOI: https://doi.org/ 10.3897/nl.40.13085

Bidzilya, O., Budashkin, Yu., Yepishin, V. 2020. Review of the tribe Anerastiini (Lepidoptera: Pyralidae: Phycitinae) from Ukraine. Zootaxa, 4718(1), 1-24. DOI: https://doi.org/10.11646/zootaxa.4718.1.1.

Bidzilya, O., Budashkin, Yu., Zhakov, A. 2016. Checklist of grass-mining moths of Ukraine with description of one new species (Lepidoptera: Elachistidae). SHILAP Revista de Lepidopterología, 44(173), 17-38. URL : https://www.redalyc.org/articulo.oa?id= 45545991003

Bidzilya, O. V., Budashkin, Yu. I., Zhakov, A. V. 2017. Checklist of scythridid moths (Lepidoptera, Scythrididae) of Ukraine with description of two new species. Zootaxa, 4291(3), 481-503. DOI: https://doi.org/10.11646/zootaxa.4291.3.3.

Bidzilya, A. V., Budashkin, Yu. I., Goloborod'ko, K. K., Demyanenko, S. A., Zhakov, A. V. 2013. New and interesting records of Microlepidoptera (Lepidoptera) from Ukraine. Contribution 2 [Новые и интересные находки микрочешуекрылых (Lеріdорtеra) в Украине. Сообщение 2]. Eversmannia [Эверсманния], 33, 23-30. URL: https://www.elibrary.ru/item.asp?id=18877890. [in Russian].

Bidzilya, A. V., Bidychak, R. M., Budashkin, Yu. I., Demyanenko, S. A., Zhakov, A. V. 2014. New and interesting records of Microlepidoptera (Lepidoptera) from Ukraine. Contribution 3 [Новые и интересные находки микрочешуекрылых (Lеріdорtеra) в Украине. Сообщение 3]. Optimization and Protection of Ecosystems [Экосистемь, их оптимизация и охрана], 11, 3-17. URL: http://nbuv.gov.ua/UJRN/ecooo_2014_11_3. [in Russian].

Budashkin, Yu. I., Bidzilya, O. V., Demyanenko, S. O. 2019. Annotated checklist of the casebearers (Lepidoptera: Coleophoridae) of Luhansk Region [Аннотированный список молей-чехлоносок (Lepidoptera: Coleophoridae) Луганской области]. The Kharkov Entomological Society Gazette [Известия Харьковского энтомологического общества], 27(1), 25-33. DOI: https://doi.org/ 10.36016/KhESG-2019-27-1-2. [in Russian].

Budashkin, Yu. I., Bidzilya, O. V., Zhakov, A. V. 2014. Case-bearer moths (Lepidoptera, Coleophoridae): Contribution to the Ukrainian fauna [Чехликовые моли (Lepidoptera, Coleophoridae): новые материалы к фауне Украины]. Ukrainska Entomofaunistyka [Українська ентомофауністика], 5(2), 1-9. URL: https://archive.org/details/UKRENTFAU521Budashkin. [in Russian].

Demyanenko, S. 2012. On the fauna of Lasiocampoidea and Bombycoidea (Insecta, Lepidoptera) of the Lugansk Region [K фayнe Lasiocampoidea и Bombycoidea (Insecta, Lepidoptera) Луганской области]. In: Zahorodniuk, I., ed. Dynamics of Biodiversity 2012 [Динаміка біорізноманіття 2012]. Taras Shevchenko National University of Luhansk, Luhansk, 212-215. ISBN: 9789666172979. URL: http://www.lepidoptera.crimea.ua/articles/Demyanenko_2012_Las_Bomb.pdf. [in Russian].

Demyanenko, S. A. 2018. A new species of noctuoid moths (Lepidoptera, Noctuoidea) for the fauna of Severodonetsk, Lugansk Region [Новые виды ноктуоидных чешуекрылых (Lepidoptera, Noctuoidea) для фауны г. Северодонецка Луганской области]. $3^{\text {rd }}$ International Scientific-Practical Conference 'Modern Problems of Natural Sciences: Theory, Practice, Educational Innovations' (dedicated to the 85 ${ }^{\text {th }}$ anniversary of the Faculty of Natural Sciences and Geography, Nizhyn, 18-19 October, 2018): proceeding [III міжнародна науково-практична конференція «Сучасні проблеми природничих наук: теорія, практика, освітні новації» (до 85-річчя природничо-географічного факультетуб Ніжин, 18-19 жовтня 2018 р.): прачі]. Nizhyn Mykola Gogol State University, Nizhyn, 58-60. URL: http://lib.ndu.edu.ua/dspace/handle/123456789/1977. [in Russian].

Demyanenko, S. O. 2019. Pyralid moths (Lepidoptera, Pyralidae) of Severodonetsk [До фауни вогнівок (Lеріdoptera, Pуralidae) Сіверодонецька]. In: Zahorodniuk, I., ed. Natural History Museology. Volume 5: Natural History Museums in Ukraine: Formation and Prospects for Development: proceedings of the scientific conference, Kyiv, October 7-8, 2019) [Природнича музеологія. Випуск 5: Природничі музеї в Украӥні: становлення та перспективи розвитку: прачі наукової конферениї, Київ, 7-8 жсовтня 2019 p.]. National Museum of Natural History, Kyiv, 159-162. ISBN: 9789660289994. URL: http://www.zoomus.lviv.ua/files/ museology5-2019_11_10_2019.pdf. [in Ukrainian].

Demyanenko, S. A., Geryak, Yu. N. 2012. The current state of the butterflies (Lepidoptera, Rhopalocera) fauna of the Lugansk region of Ukraine [Современное состояние фауны булавоусых чешуекрылых (Lepidoptera, Rhopalocera) Луганской области Украины]. Scientific Bulletin of the Uzhgorod University. Series Biology [Науковий вісник Ужгородського університету. Серія: Біологія], 33, 90-104. URL: http://nbuv.gov.ua/UJRN/Nvuu_2012_33_17. [in Russian].

Demyanenko, S. A., Severov, I. G., Kostjuk, I. Yu. 2011. On the fauna of geometrid moths (Lepidoptera, Geometridae) of the Lugansk region. [К фауне пядениц (Lepidoptera, Geometridae) Луганской области]. In: Collection of Scientific Labours of the Luhansk 
Nature Reserve - 2011 [Збірник наукових праць Луганського природного заповідника - 2011]. Luhansk Nature Reserve, Luhansk, 111-129. ISBN: 9789660262140. URL: http://www.lepidoptera.crimea.ua/articles/Demyanenko_2011_Geometridae.pdf. [in Russian].

Dubatolov, V. V., Ustjuzhanin, P. Ya., Zintshenko, V. K. 1997. A review of the Ethmiidae of the Asian part of Russia and neighbouring territories (Lepidoptera: Ethmiidae). Atalanta, 28(1/2), 161-171. URL: http://www.zobodat.at/pdf/Atalanta_28_0161-0171.pdf.

Ellis, W. N. 2021. Plant Parasites of Europe: Leafminers, Galls and Fungi. Last modified April 28, 2021. URL: https://bladmineerders.nl.

Geryak, Yu. M., Demyanenko, S. A., Zhakov, A. V., Kovalev, I. V., Kozlov, S. M., Konovalov, S. V., Mushynskii, V. G., Severov, I. G. 2012. New, little known and rare species of Noctuoidea (Insecta, Lepidoptera) for the steppe zone of Ukraine [Нові, маловідомі та рідкісні види Noctuoidea (Insecta, Lepidoptera) степової зони України]. Scientific Bulletin of the Uzhgorod University. Series Biology [Науковий вісник Ужгородського університету. Серія: Біологія], 32, 65-87. URL: https://dspace.uzhnu.edu.ua/jspui/ handle/lib/2293. [in Ukrainian].

Geryak, Yu. M., Zhakov, A. V., Kostjuk, I. Yu., Sergienko, V. M. 2014. Ecological faunistic review of Nolidae (Noctuoidea, Lepidoptera) of Ukraine [Еколого-фауністичний огляд нолід (Nolidae, Noctuoidea, Lepidoptera) фауни України]. Proceedings of the National Museum of Natural History [Вісник Національного науково-природничого музею], 12, 71-99. URL: http://nbuv.gov.ua/UJRN/ Vnnpm_2014_12_7. [in Ukrainian].

Geryak, Yu. M., Demyanenko, S. A., Konovalov, S. V., Martynov, V. V. 2015. Contribution to the knowledge of the noctuoid-moth fauna (Lepidoptera: Noctuoidea) of Donbas [До вивчення ноктуоїдних лускокрилих (Lepidoptera: Noctuoidea) Донбасу]. Scientific Principles of Biodiversity Conservation [Наукові основи збереження біотичної різноманітності], 6(1), 205-234. URL: https://ecoinst.org.ua/html/201513pdf/ts1.pdf. [in Ukrainian].

Geryak, Yu., Khalaim, E., Sergienko, V., Andrianov, O., Bezugly, S., Konovalov, S., Karmyshev, Yu., Zhakov, O., Mushynsky, V., Gerasimov, R., Tsykal, S., Trotsenko, S., Parkhomenko, V., Sheshurak, P., Bidychak, R., Demyanenko, S., Kavurka, V., Kanarsky, Yu., Kozlov, S., Kovaliov, I. 2018. New data on the species composition and distribution of Noctuoidea (Lepidoptera) of Ukraine [Нові дані про видовий склад та поширення ноктуоїдних лускокрилих (Lepidoptera: Noctuoidea) в Україні]. Ukrainska Entomofaunistyka [Украӥнська ентомофауністика], 9(3), 1-61. URL: https://drive.google.com/file/d/1ZCWddIX7D4RdgsY3JB1 Qh-_aAIpojWzo. [in Ukrainian].

Guglya, Yu. A. 2015. A review of the leafroller moths collection (Lepidoptera: Tortricidae) of the Museum of Nature of V. N. Karazin National University of Kharkiv [Обзор бабочек листоверток (Lepidoptera: Tortricidae) коллекции Музея природы Харьковского национального университета им. В. Н. Каразина]. Ukrainska Entomofaunistyka [Украӥнська ентомофауністика], 6(3), 1-21. URL: https://archive.org/details/UkrEntFau20156301Guglya. [in Russian].

Heikkilä, M., Mutanen, M., Kekkonen, M., Kaila, L. 2014. Morphology reinforces proposed molecular phylogenetic affinities: a revised classification for Gelechioidea (Lepidoptera). Cladistics, 30(6), 563-589. DOI: https://doi.org/10.1111/cla.12064.

Kavurka, V. V., Geryak, Yu. M., Demyanenko, S. A., Zaika, M. I., Nazarov, N. V., Popov, G. V., Prohorov, O. V., Novitskiy, S. M. 2018. A new records of Arachnida, Myriapoda and Insecta from Red Book of Ukraine [Нові знахідки павукоподібних (Аrасhnida), багатоніжок (Myriapoda) та комах (Insecta), занесених до Червоної Книги України]. In: Materials to $4^{\text {th }}$ Edition of Red Data Book of Ukraine. Animals. Volume 1 (Series: 'Conservation Biology in Ukraine', issue 7, volume 1) [Матеріали до 4-го видання Червоної книги Украӥни. Тваринний світ. Том. 1 (Серія: «Conservation Biology in Ukraine», випуск 7, том 1)]. I. I. Schmalhausen Institute of Zoology of the National Academy of Sciences of Ukraine, Kyiv, 276-302. ISBN 9789660285774. URL: https://uncg.org.ua/wp-content/uploads/2019/05/materialy-chku-tvarUNCG71.pdf. [in Ukrainian].

Klyuchko, Z. F., Matov, A. Yu., Severov, I. G. 2006. On the fauna of noctuids (Lepidoptera: Noctuidae s. l.) of Lugansk Area (Ukraine) [К фауне совок (Lepidoptera: Noctuidae s. l.) Луганской области (Украина)]. Eversmannia [Эверсманния], 5, 24-35. URL: https://www.elibrary.ru/item.asp?id=18835845. [in Russian].

Klyuchko, Z. F., Severov, I. G. 2006. New locations of rare species of moths (Lepidoptera: Endromidae, Sphingidae, Noctuidae, Arctiidae) in Ukraine [Нові місцезнаходження рідкісних видів лускокрилих (Lepidoptera: Endromidae, Sphingidae, Noctuidae, Arctiidae) в Україні]. Nature Reserves in Ukraine [Заповідна справа в Україні], 12(2), 67-69. URL: http://aetos.kiev.ua/zsu/zsu12-2/zsu12-214.pdf. [in Ukrainian].

Lepiforum. 2020. Ethmia vittalbella (Christoph, 1877). Version 25. Last modified November 30, 2020. URL: https://lepiforum.org/wiki/ page/Ethmia_Vittalbella.

Nieukerken, E. J. van, Kaila, L., Kitching, I. J., Kristensen, N. P., Lees, D. C., Minet, J., Mitter, C., Mutanen, M., Regier, J. C., Simonsen, T. J., Wahlberg, N., Yen, S.-H., Zahiri, R., Adamski, D., Baixeras, J., Bartsch, D., Bengtsson, B. Å., Brown, J. W., Bucheli, S. R., Davis, D. R., De Prins, J., De Prins, W., Epstein, M. E., Gentili-Poole, P., Gielis, C., Hättenschwiler, P., Hausmann, A., Holloway, J. D., Kallies, A., Karsholt, O., Kawahara, A. Y., Koster, J. C., Kozlov, M. V., Lafontaine, J. D., Lamas, G., Landry, J.-F., Lee, S., Nuss, M., Park, K.-T., Penz, C., Rota, J., Schintlmeister, A., Schmidt, B. C., Sohn, J.-C., Solis, M. A., Tarmann, G. M., Warren, A. D., Weller, S., Yakovlev, R. V., Zolotuhin, V. V. and Zwick, A. 2011. Order Lepidoptera Linnaeus, 1758. In: Zhang, Z.-Q., ed. Animal biodiversity: An outline of higher-level classification and survey of taxonomic richness, Zootaxa, 3148(1), 212-221. DOI: https://doi.org/10.11646/zootaxa.3148.1.3.

Pak, O. V., Yaroshenko, N. N. 2001. Additional matherials to pyraloid fauna (Lepidoptera: Pyraloidea) of the South-Western Ukraine [Дополнения к материалам по фауне огнёвок (Lepidoptera: Pyraloidea) Юго-Востока Украины]. Biodiversity of Natural and Man-Made Habitats of Ukraine: Proceedings of the All-Ukrainian Conference of Students, Postgraduates, and Young Scientists (Donetsk, November 19-20, 2001). Part 2 [Біорізноманіття природних i техногенних біотопів Украӥни: матеріали всеукраїнської конферениії студентів, аспірантів та молодих вчених (Донецьк, 19-20 листопада 2001 р.) Частина 2]. Donetsk National University, Donetsk, 146-152. URL: http://www.lepidoptera.crimea.ua/articles/Pak_2001_dop_Pyraloidea.djvu. [in Russian].

POWO. 2021. Plants of the World Online. Facilitated by the Royal Botanic Gardens, Kew. URL: http://www.plantsoftheworldonline.org. [Accessed: 28 April 2021].

Sattler, K. 1967. Microlepidoptera Palaearctica. Band 2. Ethmiidae. Verlag Georg Fromme \& Co, Wien, 1-185.

Sinev, S. Yu., Shovkoon, D. F. 2019. Ethmiidae. In: Sinev, S. Yu., ed. Catalogue of the Lepidoptera of Russia [Kamaлог чешуекрыльх (Lepidoptera) Poccuu]. $2^{\text {nd }}$ ed. Zoological Institute RAS, Saint Petersburg, 52-53. ISBN: 9785980920685. [in Russian].

Vasyl Karazin Kharkiv National University

Institute for Evolutionary Ecology of the National Academy of Sciences of Ukraine 


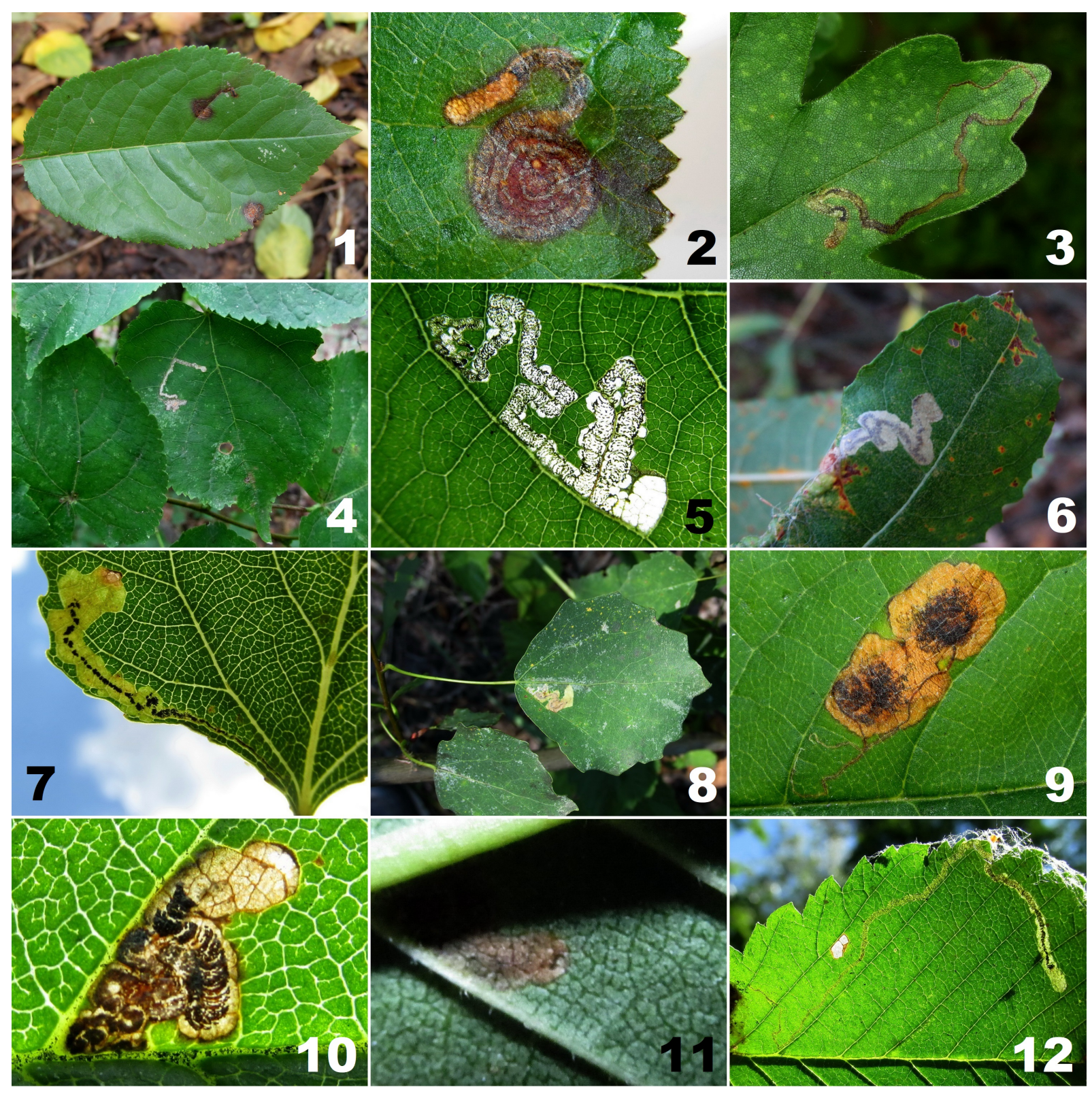

Figs. 1-12. Species of Lepidoptera new for Severodonetsk and its environs: 1-2 - Stigmella prunetorum, mines on Prunus cerasus, 28.10.2018 and 21.10.2018; 3 - Stigmella aceris, mine on Crataegus, 03.06.2018; 4-5 - Stigmella tiliae, mines on Tilia cordata, 18.07.2020 and 03.07.2020; 6 - Stigmella salicis, mine on Salix cinerea, 18.10.2020; 7 - Stigmella trimaculella, mine on Populus nigra, 13.07.2019; 8 - Stigmella assimilella, mine on Populus tremula, 07.07.2019; 9 - Stigmella plagicolella, mine on Prunus cerasus, 21.10.2018; 10-11 - Stigmella viscerella, mine and exit slit on underside of mine on Ulmus, 04.07.2019; 12 - Stigmella lemniscella, mine on Ulmus, 07.07.2019. 
S. O. DEMYANENKO, O. V. BIDZILYA, E. A. KAROLINSKIY New records of Lepidoptera (Insecta) of Severodonetsk (Luhansk Region, Ukraine) and its environs

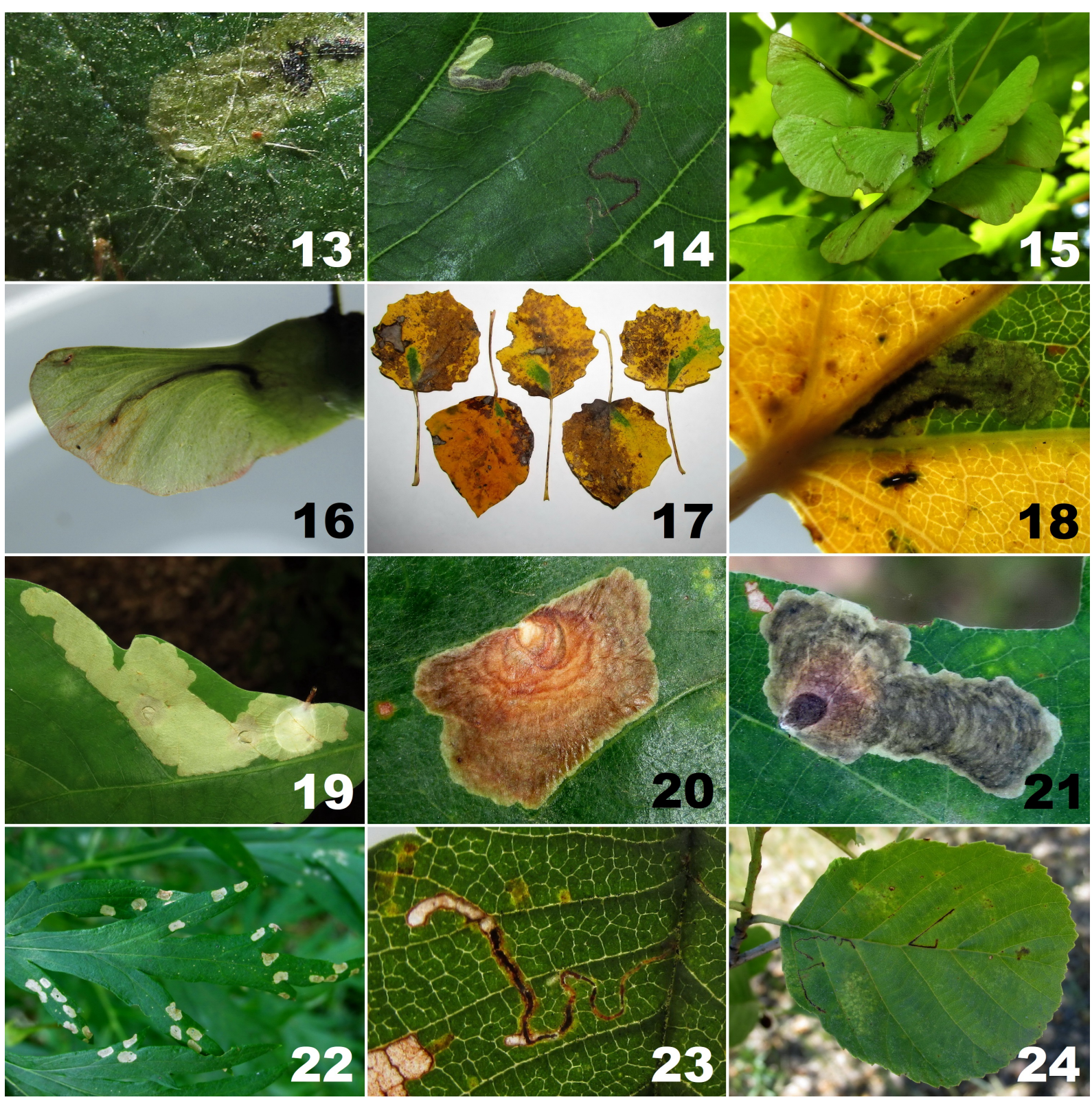

Figs. 13-24. Species of Lepidoptera new for Severodonetsk and its environs: 13- Stigmella lemniscella, exit slit on upperside of mine on Ulmus, 07.07.2019; 14 - Stigmella basiguttella, mine on Quercus robur, 08.07.2018; 15-16 - Etainia louisella, mines on Acer campestre, 07.06.2020; 17-18 - Ectoedemia argyropeza, mines on Populus tremula, 06.10.2019; 19 - Tischeria ekebladella, mine on Quercus robur, 08.07.2018; 20 — Tischeria dodonaea, mine on Quercus robur, 16.09.2018; 21 - Tischeria decidua, mine on Quercus robur, 08.09.2018; 22 - Bucculatrix noltei, secondary mines on Artemisia vulgaris leaves, 22.06.2019; 23 - Bucculatrix albedinella, mine on Ulmus, 04.07.2019; 24 - Bucculatrix cidarella, mines on Alnus glutinosa, 07.07.2019. 

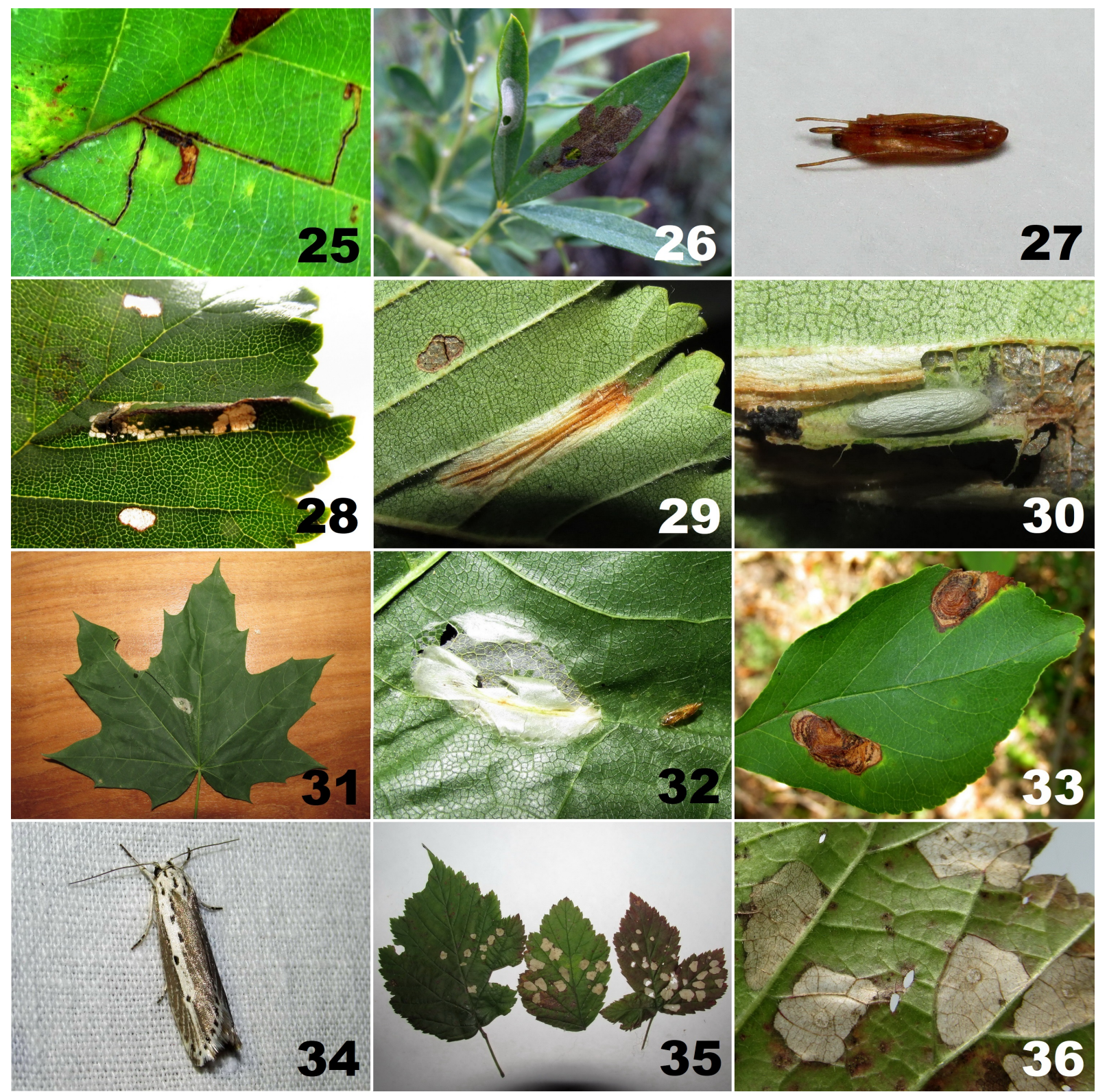

Figs. 25-36. Species of Lepidoptera new for Severodonetsk and its environs: 25 - Bucculatrix cidarella, mines on Alnus glutinosa, 07.07.2019; 26 - Micrurapteryx kollariella, mine and cocoon on Chamaecytisus, 01.08.2020; 27 - Micrurapteryx kollariella, pupa, 26.07.2020; 28-30 - Phyllonorycter schreberella, underside of mine and cocoon on Ulmus, 15.09.2019; 31-32 - Phyllonorycter joannisi, mine and underside of mine with exuvium on Acer platanoides, 27.06.2019; 33 - Leucoptera malifoliella, mines on Malus, 12.08.2018; 34 - Ethmia vittalbella, imago, 09.05.2018; 35-36 - Plegmidia potentillae, mines and underside of mines on Rubus caesius, 27.10.2019. 